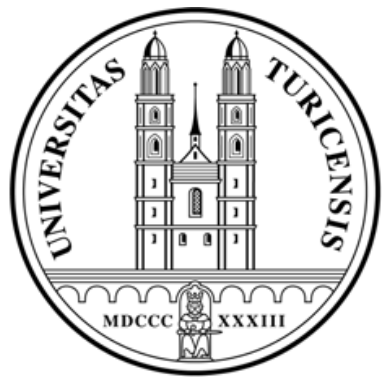

Institute for Empirical Research in Economics

University of Zurich

Working Paper Series

ISSN 1424-0459

Working Paper No. 185

Evolutionary Portfolio Selection with Liquidity Shocks

Enrico De Giorgi

May 2004 


\title{
Evolutionary Portfolio Selection with Liquidity Shocks
}

\author{
Enrico De Giorgi* \\ Institute for Empirical Economic Research \\ University of Zurich
}

First Draft: 17th December 2003

This Version: 7th May 2004

\begin{abstract}
Insurance companies invest their wealth in financial markets. The wealth evolution strongly depends on the success of their investment strategies, but also on liquidity shocks which occur during unfavourable years, when indemnities to be paid to the clients exceed collected premia. An investment strategy that does not take liquidity shocks into account, exposes insurance companies to the risk of bankruptcy, when liquidity shocks and low investment payoffs jointly appear. Therefore, regulatory authorities impose solvency restrictions to ensure that insurance companies are able to face their obligations with high probability. This paper analyses the behaviour of insurance companies in an evolutionary framework. We show that an insurance company that merely satisfies regulatory constraints will eventually vanish from the market. We give a more restrictive no bankruptcy condition for the investment strategies and we characterize trading strategies that are evolutionary stable, i.e. able to drive out any mutation.
\end{abstract}

Keywords: insurance, portfolio theory, evolutionary finance.

JEL Classification Numbers: G11, G22, D81.

SSRN Classification: Capital Markets: Asset Pricing and Valuation, Banking and Financial Institutions.

*I am grateful to Thorsten Hens and Klaus Schenk-Hoppé for their valuable suggestions. Financial supports from Credit Suisse Group, UBS AG and Swiss Re through RiskLab, Switzerland and from the national center of competence in research "Financial Valuation and Risk Management" and are gratefully acknowledged. The national centers of competence in research are managed by the Swiss National Science Foundation on behalf of the federal authorities. 


\section{Introduction}

Institutional investors, like pension plans or insurance companies, are usually active on asset markets that do not provide complete insurance against all possible risks. The performance of those institutions strongly depends on the success of their investment strategies. On the other hand, pension plans and insurance companies are also exposed to liquidity shocks, that occur when the pensions or claims to be paid out to the clients exceed collected premia. In order to ensure that insurance companies or pension plans are able to face their obligations with high probability, regulatory authorities impose solvency constraints, that are constraints on the investment strategies, such that a safely invested reserve capital exists. A part from the regulatory constraints, institutional investors still face the problem of choosing the proportion of wealth to be invested prudently, in order to be able to cover future losses and, therefore, to avoid going bankrupt, but, on the other hand, to also profit from growth opportunities offered by financial markets.

In this paper we analyse the long-run performance of insurance companies with an evolutionary model, that is well suited to study the performance of large institutional investors, since they have a considerable impact on asset prices, face relatively small transaction costs and their investment horizon is potentially infinite.

According to this approach, investors' trading strategies compete for the market capital and the endogenous price process is thus a market selection mechanism along which some strategies gain market capital while others lose. Analogously, insurance companies sell insurance contracts depending on their ability of facing liquidity shocks, and premia are also endogenously determined by demand and supply. We use the theory of dynamical systems (Arnold 1998) to derive evolutionary stable trading stable strategies, i.e. those that have the highest exponential growth rate in a population where they determine asset prices.

The evolutionary model that we present in this paper, has one long-lived risky asset and cash. Withdrawals and savings are the difference between collected premia and pensions or indemnities to be paid. Here, in particular, we consider insurance companies, and the pricing principle for insurance contracts is given by regulatory constraints, set up to ensure that with their supply for insurance contracts, insurance companies are able to face their obligations with high probability. This relates to the investment strategies, i.e. the proportion of wealth safely invested, so that, finally, regulatory constraints represent minimal requirements on investors' investment strategies. A bankruptcy occurs when the investment's payoff and premia are not enough to pay the indemnities. Moreover, borrowing and short-selling are not allowed, so investors that go to bankrupt simply disappear from the market. We establish a no bankruptcy condition for the investment strategies. The no bankruptcy condition is the minimal sufficient condition on the trading strategies that ensures that, in the presence of any type of competitor or trading strategy, the investor is able to face almost surely liquidity shocks. In fact, since asset prices are endogenously determined, it happens that, depending on other players' strategies and wealth shares, with any strategy that satisfies a less restrictive condition than the no bankruptcy condition, the probability of going bankrupt is strictly 
positive. In particular, if an investor is the unique survivor at some point in time, the no bankruptcy condition is sufficient but also necessary to avoid almost surely going bankrupt. Nevertheless, while investors with strategies satisfying the no bankruptcy condition will not almost surely go bankrupt, we also show that investors who use the simple strategy that corresponds to the no bankruptcy boundary, will eventually disappear from the market. Moreover, we characterize trading strategies that are evolutionary stable, if they exist, following the idea first introduced by Hens and Schenk-Hoppé (2002a). We give the condition on the dividend process and liquidity shocks factor for the existence of evolutionary stable strategies, when the state of the world follows an i.i.d. process. We show that the condition for the existence of evolutionary stable strategies is related to the growth rate of the trading strategies in a neighbourhood of the strategy investing according to the no bankruptcy boundary. If this growth rate is strictly positive, then an investor putting more than the no bankruptcy boundary on the risky assets is able to further increase her market share, when asset prices are dominated by the strategy corresponding to the no bankruptcy boundary. However, this is true only as long as liquidity shocks do not force the investor to use all her wealth, or else she will disappear from the market. Therefore, while it can happen that the growth rate of a strategy in a neighbourhood above the no bankruptcy boundary is positive, this strategy cannot be evolutionary stable, since it will almost surely disappear, because of liquidity shocks. In this case, no evolutionary stable strategies could exist. This result also suggest that in the presence of liquidity shocks, evolutionary stability should be characterized in term of both the growth rate and the probability of default.

This work contributes to the development of the evolutionary portfolio theory, that started with the seminal paper of Blume and Easley (1992), where an asset market model is first introduced to study the market selection mechanism and the long run evolution of investors' wealth and assets' prices. In their model, Blume and Easley (1992) consider diagonal securities $^{1}$, with no transaction costs and positive proportional saving rates are exogenously given. In the case of complete markets with diagonal securities, Blume and Easley (1992) show that there is a unique attractor of the market selection mechanism and prices do not matter. With simple strategies ${ }^{2}$ and constant, identical saving rates across investors, the unique survivor is the portfolio rule known as "betting your beliefs" (Breiman 1961), where the proportion of wealth to be put on each asset is the probability of the corresponding state of nature. This strategy can also be generated by maximizing the expected logarithm of relative returns, which is know as the Kelly rule, studied in discrete-time by Kelly (1956), Breiman (1961), Thorp (1971) and Hakansson and Ziemba (1995) (for an overview, see also Ziemba 2002) and, in continuous-time, by Pestien and Sudderth (1985), Heath, Orey, Pestien, and Sudderth (1987) and Karatzas and Shreve (1998), among others. Hens and

\footnotetext{
${ }^{1} \mathrm{~A}$ system of securities is called diagonal, if for each state of nature there is exactly one asset which has a strictly positive payoff.

${ }^{2} \mathrm{~A}$ portfolio rule called a simple strategy, if the proportion of wealth put on each asset is constant over time.
} 
Schenk-Hoppé (2002a) proposed a more general setting, with incomplete markets, general short-lived assets that re-born each period and constant, positive, proportional and, identical saving rates across investors. In their evolutionary model, the equilibrium notion refers to wealth distributions that are invariant under the market selection process. The authors show that invariant wealth distributions are generated by a population, where only one investor (or portfolio rule) exists (a so-called monomorphic population). Moreover, they introduce the concept of evolutionary stable portfolio rules, that is also considered in this paper. The main result of Hens and Schenk-Hoppé (2002a) is that, in the case of ergodic state of the world processes and without redundant assets, there is a unique evolutionary stable portfolio rule, which is the one that puts on each asset the proportion of wealth corresponding to the expected relative payoff of the asset. In Evstigineev, Hens, and Schenk-Hoppé (2003) this result is extended to a model with long-lived assets, under the assumption of Markow state of the world. Introducing long-lived assets allows to take into account the capital gains and losses due to assets' prices changes. This will also be of much importance in the presence of liquidity shocks, as we will discuss in this paper. Moreover, in Evstigneev, Hens, and Schenk-Hoppé (2002) it is also shown that, with independent and identically distributed state of world processes, the strategy that invests according to relative dividends is the unique simple portfolio rule that asymptotically gathers total wealth. A generalization of the results obtained by Blume and Easley (1992). Sandroni (2000), and Blume and Easley (2002) have also studied the case of long-lived assets, to include market prices in the evolution of wealth shares. The main result of Blume and Easley (2002) and Sandroni (2000), is that, with complete markets, among all infinite horizon expected utility maximizers, those who happen to have rational expectation will eventually dominate the market and this result holds independently of investors' risk aversion. In his model Sandroni (2000) also includes endogenously determined positive and proportional saving rates.

All these models assume that withdrawals and savings are a positive proportion of the current wealth, so that bankruptcy is excluded in their setup. Moreover, e.g. in Evstigineev, Hens, and Schenk-Hoppé (2003), the withdrawal rates are assumed to be identical among investors. Under these assumptions, the only criterion that matters for a trading strategy to be evolutionary stable, is its exponential growth rate in the presence of a mutant strategy. This paper shows that with non-proportional and maybe negative withdrawal rates, a second criterion has to be considered, since in fact, even if a strategy has the maximal exponential growth rate in the presence of any mutant, it can disappear because exogenously determined liquidity shock occurs.

In the classical finance approach with exogenously given price dynamics, asset-liability management models already assume that investors maximize the investment's expected payoff less penalties for bankruptcy or targets not meet (see Carino, Myers, and Ziemba 1998, Carino and Ziemba 1998). Liu, Longstaff, and Pan (2003) consider a price dynamic for the risky asset with jumps (event risk) and take utility functions identical to $-\infty$ for strictly negative terminal wealth, so that no portfolio rule, that has a strictly positive probability 
of going bankrupt, will be optimal. They obtain lower (since they do not exclude shortselling) and upper bounds for the proportion of wealth to be put on the risky asset and they provide optimal portfolio weights. Alternatively, Browne (1997) distinguishes between the survival problem and the growth problem. He first looks at portfolio rules that maximize the probability of surviving in the so-called danger-zone (where bankruptcy has strictly positive probability to occur) and second, he considers portfolio rules that maximize the growth rate in the safe-zone, where bankruptcy is almost surely excluded. Browne (1997) identifies wealth-level dependent strategies, but in his time-continuous setup, no optimal strategy is found for the danger-zone, and a weaker optimality criterion is introduced. The optimal strategy for the safe-zone corresponds to a generalization of the Kelly criterion previously discussed. Zhao and Ziemba (2000) propose a model with a reward function on minimum subsistence, i.e. the objective function to maximize equals the sum of the expected final wealth and a concave increasing function on the supremum over the wealth levels that are almost surely smaller than final wealth. In this way, the optimal portfolio rule solves a trade-off between expected payoff and minimum subsistence.

The rest of this paper is organized as follows. In the next section we present the model setup. In Section 3 we derive the no bankruptcy condition on investment strategies, that ensures that liquidity shocks do not cause bankruptcy. In Section 4 we present the main results of the paper. Section 5 concludes. Technical results and proofs are given in the Appendix.

\section{An evolutionary model with bankruptcy}

Time is discrete and denoted by $t=0,1,2, \ldots$ Uncertainty is modelled by a stochastic process $\left(S_{t}\right)_{t \in \mathbb{Z}}$ with values in some infinite space $\mathcal{S}$, endowed with power $\sigma$-algebra $2^{|S|}$. $\mathcal{F}^{t}=\sigma\left(\ldots, S_{0}, S_{1}, \ldots, S_{t}\right)$ denotes the $\sigma$-algebra giving all the information available at time $t$ and $\mathcal{F}=\sigma\left(\cup_{t \in \mathbb{Z}} \mathcal{F}^{t}\right)$. Let $\Omega=S^{\mathbb{Z}}$ be the space of sample paths $\left(s_{t}\right)_{t \in \mathbb{Z}}$, where $s_{t}, t \in \mathbb{Z}$ is the realization of $S_{t}$ on $\mathcal{S}$. Finally, $\mathbb{P}$ denotes the unique probability measure on $(\Omega, \mathcal{F})$ generated by $\left(S_{t}\right)_{t \in \mathbb{Z}}$. There are $i=1, \ldots, I(I \geq 2)$ investors, with initial wealth $w_{0}^{i}$.

There is one long-lived risky asset and cash. Cash is risk-less both in terms of its return $R=1+r \geq 1$ and price, which is taken as numéraire. The risky asset pays a dividend $D_{t}\left(s^{t}\right) \geq 0$ at time $t$, depending on the history $s^{t}=\left(\ldots, s_{-1}, s_{0}, s_{1}, \ldots, s_{t}\right)$ up to time $t$. Moreover, at each time $t$ each investor $i$ withdraws or collects the amount $C_{t}^{i}\left(s^{t}\right)$, also depending on the history $s^{t}$ up to time $t$. Here, we consider insurance companies, so that $C_{t}^{i}$ is the difference between the indemnities to be paid and collected premia.

Let $w_{t}^{i}$ be the total wealth of investor $i$ at time $t$ after claims' payment and premia collection, $m_{t}^{i} \geq 0$ and $a_{t}^{i} \geq 0$ be the unit of cash and risky asset, respectively, held by investor $i$ at time $t$ and $q_{t}$ be the price of the risky asset. The budget constraint at time $t$ of each investor 
$i$ is given by

$$
w_{t}^{i}=m_{t}^{i}+q_{t} a_{t}^{i} .
$$

The wealth of investor $i$ evolves as follows ${ }^{3}$

$$
w_{t+1}^{i}=(1+r) m_{t}^{i}+\left(D_{t+1}+q_{t+1}\right) a_{t}^{i}-C_{t+1}^{i} .
$$

We say that investor $i$ goes bankrupt during period $(t, t+1$ ] (or simply period $t+1$ ) iff $w_{t+1}^{i} \leq 0$. In this case she uses all her wealth to pay the indemnities and vanishes from the market, i.e. we arbitrarily write $m_{s}^{i}=a_{s}^{i}=0$ for all $s \geq t+1$ (and thus we also set $w_{s}^{i}=0$ for all $s \geq t+1$ ). Note that the investor's wealth at time $t+1$ also depends on the price $q_{t+1}$ of the risky asset, which is determined at equilibrium by investors' demand for the risky asset and supply. Thus, time $t+1$ investors' strategies may cause a bankruptcy. Let $\mathcal{I}_{t}=\left\{i \mid w_{t}^{i}>0\right\}$ be the set of investors, who survive period $t$. Obviously, $\mathcal{I}_{t} \subseteq \mathcal{I}_{t-1}$ and thus $m_{t}^{i}=a_{t}^{i}=0$ for all $i \notin \mathcal{I}_{t-1}$. Investor $j$ is said to be the unique survivor at time $t$ if and only if $\mathcal{I}_{t}=\{j\}$.

The next period amount $C_{t+1}^{i}$ is determined by the following. At time $t$ investor $i$ can decide to sell $\delta_{t}^{i} \geq 0$ insurance contracts on one single future stochastic claim $X_{t+1} \geq 0$ (which is identical for all investors). The premium $P_{t+1}$ of each contract is $\mathcal{F}^{t}$-measurable (depends only on information available up to time $t$ ), determined by the market clearing condition on the insurance market at time $t$, and is paid at time $t+1$ by the buyer of the insurance contract, who is supposed to be external to the economy just defined, i.e. buyers of insurance contracts do not participate to the financial market. The amount collected or withdrawn by investor $i$ at time $t+1$ is then given by the difference

$$
C_{t+1}^{i}=\delta_{t}^{i}\left(X_{t+1}-P_{t+1}\right)
$$

between claims and premia.

We suppose that the insurance market is regulated and solvency constraints are imposed. Each investor $i$ should be able to meet her obligation, in a way that conditioning on the current history $s^{t}$ only a proportion $\alpha_{t}^{i}>0$ of her current wealth will be affected with a small probability $\epsilon_{t}^{i}>0$, i.e.

$$
\mathbb{P}\left[\delta_{t}^{i}\left(X_{t+1}-P_{t+1}\right)>\alpha_{t}^{i} w_{t}^{i} \mid s^{t}\right]=\epsilon_{t}^{i}
$$

where for all $t$ and $i \in \mathcal{I}_{t}$

$$
\alpha_{t}^{i} \in(0, \bar{\alpha}) \quad \text { and } \quad \epsilon_{t}^{i} \leq \epsilon
$$

${ }^{3}$ To be formally correct, the wealth evolution of equation (2) should be replaced by

$$
w_{t+1}^{i}=\left[(1+r) m_{t}^{i}+\left(D_{t+1}+q_{t+1}\right) a_{t}^{i}-C_{t+1}^{i}\right]^{+},
$$

where for $x \in \mathbb{R}, x^{+}=\max (0, x)$. We prefer to keep the notation simpler and since we are essentially looking at strategies that survive in the long run, the wealth evolution of those strategies is correctly given by equation (2). 
The parameters $\bar{\alpha}$ and $\epsilon$ are exogenously given by regulatory authorities. Equation (3) defines the pricing rule for insurance contracts and is called quintile principle and it has been discussed in Schnieper (1993) and Embrechts (1996). Moreover, it corresponds to the proportional value-at-risk constraints studied by Leippold, Vanini, and Trojani (2003) with time independent proportional factors, and sameness between investors in their general equilibrium consideration. The parameters $\alpha_{t}^{i}$ and $\epsilon_{t}^{i}$ are fixed and can be interpreted as the "loss acceptability" of investor $i$ and, in the general setting of the model, we assume that they can vary between investors. Other simplifying assumptions will be introduced later. For a given premium $P_{t+1}$ and parameters $\alpha_{t}^{i}$ and $\epsilon_{t}^{i}$, equation (3) serves to compute the number $\delta_{t}^{i}$ of insurance contracts that investor $i$ can sell, in order to satisfy the solvency constraint. The premium $P_{t+1}$ is determined endogenously when the insurance market clears.

We should bear in mind that for an investor, going bankrupt means vanishing from the market and thus should be avoided! They can further decrease their insurance risk by choosing a smaller $\alpha^{i}$ or a smaller $\epsilon^{i}$. As we will see below, an investor with a small $\alpha^{i}$, who is a "safer investor" with respect to minimal solvency requirement, is also forced to reduce her exposure to the insurance market, "losing" in this way growth opportunities when claims are less than premia. The amount $\alpha_{t}^{i} w_{t}^{i}$ represents the technical reserve or the proportion of current wealth to be invested prudently by investor $i$ to make the risky insurance business acceptable in the future (see Norberg and Sundt 1985). If the amount $\delta_{t}^{i}\left(X_{t+1}-P_{t+1}\right)$ is strictly greater than the technical reserves, we say that investor $i$ faces a liquidity shock. From equation (3), investor $i$ faces liquidity shocks with probability $\epsilon_{t}^{i}$ during period $t+1$. In our setting, the solvency constraint essentially imposes that $m_{t}^{i} \geq \frac{\alpha_{t}^{i}}{R} w_{t}^{i}$, for all $i$, or equivalently

$$
\frac{m_{t}^{i}}{w_{t}^{i}} \geq \frac{\alpha_{t}^{i}}{R}, \quad \forall i \in \mathcal{I}_{t}
$$

Let $\mu_{t}=\mathbb{E}\left[X_{t} \mid \mathcal{F}^{t-1}\right]$ and $\sigma_{t}^{2}=\operatorname{Var}\left(X_{t} \mid \mathcal{F}^{t-1}\right)$ be the conditional expectation and the conditional variance of $X_{t}$, given $\mathcal{F}^{t}$, respectively, and let $F_{t}$ be the conditional cumulative distribution function of $Y_{t}=\frac{X_{t}-\mu_{t}}{\sigma_{t}}$, i.e.

$$
F_{t}(y)=\mathbb{P}\left[Y_{t} \leq y \mid \mathcal{F}^{t-1}\right] .
$$

Moreover, $F_{t}^{-1}$ denotes the generalized inverse of $F_{t}$. To avoid the premium $P_{t+1}$ fully covering the insurance risk, we impose the following restrictions

Assumption 1 (Insurance market). For $t \in \mathbb{Z}$ and $i \in \mathcal{I}_{t}$, let $\left(\alpha_{t}^{i}, \epsilon_{t}^{i}\right)$ and $\left(\tilde{\alpha}_{t}^{i}, \tilde{\epsilon}_{t}^{i}\right)$ be two possible choices for the loss acceptability parameters of investor $i$. Let $\alpha_{t}^{i}=\tilde{\alpha}_{t}^{i}$. Then for all premia $P_{t+1}$

$$
\delta_{t}^{i}>\tilde{\delta}_{t}^{i} \Rightarrow \epsilon_{t}^{i}>\tilde{\epsilon}_{t}^{i}
$$

This assumption says that for given technical reserves, the probability of having liquidity shocks strictly increases with the number of insurance contracts sold. If this is not satisfied, 
then it would be possible to cover additional insurance risk only through collected premia, which is not a fair pricing rule. Since $\delta_{t}^{i}=0$ solves equation (3) with $\alpha_{t}^{i}=0$ and $\epsilon_{t}^{i}=0$, Assumption 1 also implies that an insurance company without technical reserves that sells a strictly positive number of contracts, faces liquidity shocks with a strictly positive probability. Assumption 1 indirectly imposes restrictions on equilibrium premia, as shown in the following lemma.

Lemma 1. If Assumption 1 holds, then for all $t \in \mathbb{Z}$ and $i \in \mathcal{I}_{t}$ :

$$
P_{t+1}<\mu_{t+1}+\sigma_{t+1} F_{t+1}^{-1}\left(1-\epsilon_{t}^{i}\right) .
$$

Proof. Let us suppose that

$$
P_{t+1} \geq \mu_{t+1}+\sigma_{t+1} F_{t+1}^{-1}\left(1-\epsilon_{t}^{i}\right)
$$

for some $t$ and $i \in \mathcal{I}_{t}$. Then

$$
\frac{P_{t+1}-\mu_{t+1}}{\sigma_{t+1}} \geq F_{t+1}^{-1}\left(1-\epsilon_{t}^{i}\right)
$$

and thus for all $\delta>0$

$$
\mathbb{P}\left[\delta\left(X_{t+1}-P_{t+1}\right)>0\right]=\mathbb{P}\left[X_{t+1}-P_{t+1}>0\right]=\mathbb{P}\left[Y_{t+1}>\frac{P_{t+1}-\mu_{t+1}}{\sigma_{t+1}}\right] \leq \epsilon_{t}^{i}
$$

independently from $\delta$. This contradicts Assumption 1, since the last inequality shows that the probability of liquidity shocks is in fact independent of the number of contracts, with fixed technical reserves.

From equation (3) and Lemma 1, we obtain

$$
\delta_{t}^{i}\left[\mu_{t+1}+\sigma_{t+1} F_{t+1}^{-1}\left(1-\epsilon_{t}^{i}\right)-P_{t+1}\right]=\alpha_{t}^{i} w_{t}^{i},
$$

or

$$
\delta_{t}^{i}=\frac{\alpha_{t}^{i} w_{t}^{i}}{\mu_{t+1}+\sigma_{t+1} F_{t+1}^{-1}\left(1-\epsilon_{t}^{i}\right)-P_{t+1}} .
$$

Lemma 1 ensures that $\delta_{t}^{i} \geq 0$. Equation (7) says that investor $i$ supply for insurance contracts is proportional to her technical reserve and decreases with increasing probability $\epsilon_{t}^{i}$. For a fixed supply of insurance contracts, an investor can therefore decrease her technical reserve by decreasing her liquidity shock probability $\epsilon_{t}^{i}$. Naturally, the solvency constraints (3) and (4) do not take into account the magnitude of a liquidity shock! This is a well know critique of quintile constraints (see e.g. Artzner, Delbaen, Eber, and Heath 1997).

We assume that demand for insurance contracts is normalized to 1, i.e. $\sum_{i} \delta_{t}^{i}=1$ for all $t$. It follows:

$$
P_{t+1}=\mu_{t+1}+\sigma_{t+1} \sum_{i} \delta_{t}^{i} F_{t+1}^{-1}\left(1-\epsilon_{t}^{i}\right)-\sum_{i} \alpha_{t}^{i} w_{t}^{i}
$$


$\sigma_{t+1} \sum_{i} \delta_{t}^{i} F_{t+1}^{-1}\left(1-\epsilon_{t}^{i}\right)-\sum_{i} \alpha_{t}^{i} w_{t}^{i}$ is the so-called loading factor and is supposed to be strictly positive. In fact, it is well known from the ruin theory, that if $P_{t+1} \leq \mu_{t+1}$, i.e. if the premium at time $t$ is less or equal to the conditional expectation of next period claims given all information available at time $t$, then for any value for the initial wealth (without financial market) the probability of going bankrupt is equal one (see Feller 1971, page 396). From the last equation, we see that the premium of the insurance contract increases with increasing conditional variance, as one would expect, and decreases when the weighted wealth $\sum_{i=1}^{I} \alpha_{t}^{i} w_{t}^{i}$ increases. Moreover, a safer investor, with a smaller $\alpha^{i}$ or a smaller $\epsilon^{i}$ than a riskier investor, contributes to an increase of the premium, from which all investors benefit. This behaviour has also been described by Ceccarelli (2002). Equations (7) and (8) can be solved for $\delta_{t}^{i}$ and $P_{t+1}$ : they provide a unique solution with a strictly positive premium (this will become clear for the special case considered below; however, we give a general proof of the existence and uniqueness of a solution in the Appendix 6.1).

Since the goal of this paper is to analyse investors' long-run wealth evolution with respect to their investment strategies on financial markets, we assume that their profiles on insurance markets are identical, meaning that they possess the same loss acceptability parameters. Here, we do not address the question of investors' strategies (choice of the loss acceptability parameters) on the insurance market. It is not clear whether an investor who has higher loss acceptability, will growth faster or not. In fact, while it is true by equation (7) that higher loss acceptability means greater liquidity shocks (for both the probability and the amount), it must also be said that investors who sell a larger number of contracts benefit from growth opportunities when premia are greater than claims. Moreover, less technical reserves means a smaller exposure to liquidity shocks (as discussed above), but also less restrictive constraints for the investment strategies, meaning that those investors can put less money into the risk-free asset and profit from growth opportunities on the financial market. We address these issues in other works. Here, as in Leippold, Vanini, and Trojani (2003) we make the following assumption.

\section{Assumption 2 (Loss acceptability).}

Investors' "loss acceptability" is constant over time and identical for all investors, i.e. $\alpha_{t}^{i}=$ $\alpha \in(0, \bar{\alpha})$ and $\epsilon_{t}^{i}=\epsilon$ for all $t$ and $i=1, \ldots, I$.

Note that by equations (7) and (8), when all investors possess the same $\epsilon_{t}^{i}, \delta_{t}^{i}$ does not depend on $\epsilon_{t}^{i}$ anymore and therefore the magnitude of liquidity shocks is minimized for all investors if $\epsilon_{t}^{i}=\epsilon$. Moreover, by Assumption 2 and equations (7) and (8) it follows

$$
\begin{aligned}
P_{t+1} & =\mu_{t+1}+\sigma_{t+1} F_{t+1}^{-1}(1-\epsilon)-\alpha \sum_{i} w_{t}^{i}, \\
\delta_{t}^{i} & =\frac{w_{t}^{i}}{\sum_{j} w_{t}^{j}}
\end{aligned}
$$


and therefore investor's $i$ supply for insurance contracts corresponds to her relative wealth.

Now, we introduce a precise structure for the claim $X_{t+1}$. In particular, we assume that the total claim $X_{t+1}$ is proportional to the aggregate wealth available at time $t$, meaning that the amount of insured claims increases or decreases depending on the aggregate success of the investors (a similar assumption will be also made for the dividend process). This assumption also prevents a shock from destroying the economy. The proportional factor is supposed to be independent of the history up to time $t$ and can be interpreted as the liquidity shock factor for the economy. Mathematically we have

$$
X_{t+1}=\eta_{t+1} W_{t},
$$

where $\eta_{t+1} \in[0,1]$ is independent of $\mathcal{F}^{t}$ and $W_{t}=\sum_{i \in \mathcal{I}_{t}} w_{t}^{i}=\sum_{i=1}^{I} w_{t}^{i}$ is the aggregate wealth available in the economy at time $t$. From equation (11) it follows that $\mu_{t+1}=W_{t} \mathbb{E}\left[\eta_{t+1}\right]$ and $\sigma_{t+1}^{2}=W_{t}^{2} \operatorname{Var}\left(\eta_{t+1}\right)$. Moreover, $\eta_{t+1} \sim G_{t+1}$ where $F_{t+1}(y)=G_{t+1}\left(\frac{y}{W_{t}}\right), \forall y$. Thus

$$
P_{t+1}=\left(\mu\left(\eta_{t+1}\right)+\sigma\left(\eta_{t+1}\right) G_{t+1}^{-1}(1-\epsilon)-\alpha\right) W_{t} .
$$

Therefore, the premium $P_{t+1}$ is strictly positive for all $t$, if the loading factor $\left(\sigma\left(\eta_{t+1}\right) G_{t+1}^{-1}(1-\right.$ $\epsilon)-\alpha) W_{t}$ is greater than zero for all $t$. Moreover, for the sake of simplicity, we make the following assumption:

\section{Assumption 3 (Liquidity shocks).}

Liquidity shocks $\left(\eta_{t}\right)_{t \geq 1}$ are independent and identically distributed, i.e. $G_{t}=G$ for all $t$, $\eta_{t} \sim \eta \sim G$, where $G$ is a continuous cumulative distribution function.

Let $\mu=\mathbb{E}[\eta]$ and $\sigma^{2}=\operatorname{Var}(\eta)$, then by Assumptions 2 and 3 ,

$$
\begin{aligned}
& P_{t+1}=\mu W_{t}+\sigma G^{-1}(1-\epsilon) W_{t}-\alpha W_{t}=(\beta-\alpha) W_{t}, \\
& C_{t+1}^{i}=\left(\eta_{t+1}-\beta+\alpha\right) w_{t}^{i},
\end{aligned}
$$

where $\beta=\mu+\sigma G^{-1}(1-\epsilon)$. As discussed above for the general case, we impose that the loading factor $\left(\sigma G^{-1}(1-\epsilon)-\alpha\right) W_{t}$ is strictly positive, i.e. $\alpha<\min \left\{\bar{\alpha}, \sigma G^{-1}(1-\epsilon)\right\}$. Then $\beta-\alpha>\beta-\sigma G^{-1}(1-\epsilon)=\mu>0$ and thus $P_{t+1}>0$ for all $t$.

We now turn our attention to the financial market. We suppose that the risky asset is in fixed supply, normalized to one. Instead, the supply of cash is exogenously given by cumulated dividends and collected premia less withdrawals. The market clearing conditions are

$$
\begin{aligned}
\sum_{i=1}^{I} a_{t}^{i} & =\sum_{i \in \mathcal{I}_{t}} a_{t}^{i}=1 \\
M_{t} & =R \sum_{i \in \mathcal{I}_{t}} m_{t-1}^{i}+D_{t} \sum_{i \in \mathcal{I}_{t}} a_{t-1}^{i}-q_{t}\left(1-\sum_{i \in \mathcal{I}_{t}} a_{t-1}^{i}\right)-C_{t}^{i}
\end{aligned}
$$


where $M_{t}=\sum_{i \in \mathcal{I}_{t}} m_{t}^{i}$ and $C_{t}=\sum_{i \in \mathcal{I}_{t}} C_{t}^{i}$. Note that $\sum_{i \in \mathcal{I}_{t}} m_{t-1}^{i} \leq M_{t-1}=\sum_{i \in \mathcal{I}_{t-1}} m_{t-1}^{i}$ since $\mathcal{I}_{t} \subseteq \mathcal{I}_{t-1}$. Moreover, if no bankruptcy occurs during period $t$, then $\mathcal{I}_{t}=\mathcal{I}_{t-1}$ and the usual equation for $M_{t}$ follows, i.e. $M_{t}=R M_{t-1}+D_{t}-C_{t}$. Note that $M_{t} \geq 0$ for all $t$. In fact if for some $t, M_{t}<0$, then there exists at least one investor, say $j \in \mathcal{I}_{t}$, with $m_{t}^{j}<0$. But since borrowing is not allowed, investor $j$ is forced into bankruptcy during period $t$, a contradiction to $j \in \mathcal{I}_{t}$.

To be consistent with Assumption 3, and in order to avoid that dividends become very small as compared to insurance shocks, we make the following assumption for the dividend process:

\section{Assumption 4 (Dividend process).}

(i) For each $t$,

$$
D_{t}=d_{t} W_{t-1}
$$

for some process $\left(d_{t}\right)_{t>0}$, with $d_{t} \sim d \sim H$ independently and identically distributed with cumulative distribution function $H$ on $[0,1]$.

(ii)

$$
\mathbb{P}[d>0]=1-H(0) \in(0,1),
$$

i.e. at each time dividends have strictly positive probability of being zero and of being strictly positive.

This assumption, together with Assumption 3, solves the difficulty encountered by Hens and Schenk-Hoppé (2002b), where the rate of return on the long-lived asset eventually dominates that of the numéraire, so that the strategy that invests only in long-lived asset is able to drive out any other strategy. Hens and Schenk-Hoppé (2002b) suggest to base evolutionary finance model on Lucas (1978), where assets' payoffs are in term of a single perishable consumption good. In this way, the consumption rate is at least as the growth rate of the total payoff of the market. In our model, also without relaying on Lucas (1978), the pricing rule for insurance contracts (that also determines $C_{t}$ ) and, Assumption 3 and 4, ensure that the rate of "consumption" increases proportionally to the growth rate of the total payoff. Moreover, as will discuss later, if assets' payoffs were in term of perishable consumption goods, it would not be possible to find a trading strategy that preserves the wealth (the reserve capital in the insurance business) and have positive growth rate.

Let $\lambda_{t}^{i} \in[0,1]$ be the proportion of wealth invested in the risky asset by investor $i \in \mathcal{I}_{t}$ at time $t$. We have

$$
a_{t}^{i}=\frac{\lambda_{t}^{i} w_{t}^{i}}{q_{t}} \quad \text { and } \quad m_{t}^{i}=\left(1-\lambda_{t}^{i}\right) w_{t}^{i}
$$

We call the sequence $\left(\lambda_{t}^{i}\right)_{\left\{t>0 \mid i \in \mathcal{I}_{t}\right\}}$ the trading strategy of investor $i$ and $\lambda_{t}^{i}$ the strategy of investor $i$ at time $t$. We use the convention that $\lambda_{t}^{i}=0$ if $i \notin \mathcal{I}_{t}$. Note that $\lambda_{t}^{i}$ is a random variable, i.e. it depends on the state of the world up to time $t, s^{t}$. Other assumptions on the 
process defining the trading strategy $\left(\lambda_{t}^{i}\right)_{t \geq 0}$ will be introduced later. Here, we just impose the following restriction on the strategies at time $t,\left(\lambda_{t}^{i}\right)_{i \in \mathcal{I}_{t}}$, to prevent the price of the risky asset from becoming zero.

\section{Assumption 5 (Investors' strategies).}

For each $t$ such that $\left|\mathcal{I}_{t}\right|>1$, there exists $i, j \in \mathcal{I}_{t}$ with $\left(1-\lambda_{t}^{i}\right) \lambda_{t}^{j}>0$.

Assumption 5 essentially states that if more than one investor survives period $t$, then there exists at least one survivor with a strictly positive proportion of her wealth invested in the risky asset and one survivor with a strictly positive proportion of her wealth invested in the risk-free asset. Naturally, when a survivor has a mixed strategy ${ }^{4} \lambda_{t}^{i} \in(0,1)$, then Assumption 5 is obviously satisfied with $i=j$. If $\left|\mathcal{I}_{t}\right|=1$, then it might occur that the unique survivor uses a strategy investing all her wealth in the risk-free asset. The strategy $\lambda_{t}^{i}=1$ is excluded by the solvency constraint. In fact, the solvency condition stated by equation (5) is equivalent to

$$
1-\lambda_{t}^{i} \geq \frac{\alpha}{R} \Leftrightarrow \lambda_{t}^{i} \leq 1-\frac{\alpha}{R}=: \bar{\lambda} \in(0,1),
$$

i.e., for each investor, the proportion of wealth invested in the risky asset is bounded from above by $\bar{\lambda}$. It seems to be a natural restriction for an insurance company (or a pension fund), as shown e.g. in Davis (2001, Tables 5 and 6) for life insurances and pension funds of several countries. Let $\boldsymbol{\lambda}_{t}=\left(\lambda_{t}^{1}, \ldots, \lambda_{t}^{I}\right)^{\prime}$, then the market clearing condition for the risky asset (14) implies

$$
q_{t}=\boldsymbol{\lambda}_{t}^{\prime} \mathbf{w}_{t}
$$

Note that for $i \notin \mathcal{I}_{t}, w_{t}^{i}=0$ by assumption and thus $\lambda_{t}^{\prime} \mathbf{w}_{t}=\sum_{i \in \mathcal{I}_{t}} \lambda_{t}^{i} w_{t}^{i}$. We rewrite equation (2) as follows

$$
w_{t+1}^{i}=\left[R\left(1-\lambda_{t}^{i}\right)+\left(d_{t+1} W_{t}+q_{t+1}\right) \frac{\lambda_{t}^{i}}{q_{t}}-\left(\eta_{t+1}-\beta+\alpha\right)\right] w_{t}^{i} .
$$

\section{The no bankruptcy condition}

Before discussing the long run wealth evolution of investment strategies, we give conditions for avoiding bankruptcy. In fact, a necessary condition for long-term survival is not to go bankrupt and strategies that do not almost surely exclude bankruptcy are avoided by investors with long-term horizon. Therefore, as in Browne (1997) and Liu, Longstaff, and Pan (2003), we distinguish between the conditions on the strategies to avoid going bankrupt and

\footnotetext{
${ }^{4} \mathrm{~A}$ strategy $\left(\lambda_{t}^{i}\right)_{t}$ is called a mixed strategy, iff it assigns a strictly positive percentage to every asset, for all $t$. In our setting, a mixed strategy is characterized by $\lambda_{t}^{i} \in(0,1)$ for all $t$ (see Evstigneev, Hens, and Schenk-Hoppé 2002).
} 
then, given that investors satisfy those conditions, we analyses the long-term wealth evolution. In our setting, analogously to Liu, Longstaff, and Pan (2003), we obtain upper bounds for the $\lambda_{t}^{i}$ s (a lower bound is given by the no short sale restriction). We will show below that an investor with a strategy that does not prevent bankruptcy at each period, has a strictly positive probability of vanishing from the market, even if she is the unique survivor. Moreover, if an investor uses a simple strategy that does not prevent bankruptcy, she has probability 1 of vanishing from the market, even if at some point in time she is the unique survivor and thus dominates assets' prices. In particular, an investor holding only the risky asset (i.e. $\lambda_{t}^{i}=1$ ) becomes extinct with probability 1 . This result shows that Theorem 1 in Hens and Schenk-Hoppé (2002b) does not hold when bankruptcy can occur.

We first consider the case $\left|\mathcal{I}_{t}\right|=1$ for some $t>0$, i.e. $\mathcal{I}_{t}=\{j\}$ for some $j \in\{1, \ldots, I\}$. We restrict ourself to strategies $\lambda_{t}^{j}>0$. If $\lambda_{t}^{j}=0$, as is clearly excluded since $R>$ $\sup \operatorname{supp}(\eta)-\beta+\alpha^{5}$ ! The price of the risky asset at time $t$ is given by $q_{t}=\lambda_{t}^{j} w_{t}^{j}$ and the aggregate wealth at time $t$ is $W_{t}=w_{t}^{j}$ : from equation (17) it follows immediately that

$$
j \in \mathcal{I}_{t+1} \Leftrightarrow R\left(1-\lambda_{t}^{j}\right)-\left(\eta_{t+1}-\beta+\alpha\right)+d_{t+1}>0 .
$$

Let $\eta=\inf \operatorname{supp}(\eta), \bar{\eta}=\sup \operatorname{supp}(\eta)$ and $\bar{d}=\sup \operatorname{supp}(d)$ and $K$ be the continuous multivariate cumulative distribution of $(\eta, d)$ on $[\underline{\eta}, \bar{\eta}] \times[0, \bar{d}]$, i.e.

$$
K(x, y)=\mathbb{P}[\eta \leq x, d \leq y]
$$

Moreover, let $\tilde{K}(z)=\mathbb{P}[d-\eta \leq z]=\int_{\underline{\eta}}^{\bar{\eta}} \int_{0}^{x+z} d K(x, y)$ be the cumulative distribution function of $d-\eta$. Then

$$
\begin{aligned}
\mathbb{P}\left[j \in \mathcal{I}_{t+1}\right] & =\mathbb{P}\left[d_{t+1}-\eta_{t+1}>-R\left(1-\lambda_{t}^{j}\right)-\beta+\alpha\right] \\
& =1-\tilde{K}\left(-R\left(1-\lambda_{t}^{j}\right)-\beta+\alpha\right)
\end{aligned}
$$

and thus

$$
\mathbb{P}\left[j \in \mathcal{I}_{t+1}\right]=1 \Leftrightarrow \lambda_{t}^{j} \leq \frac{R+\underline{k}+\beta-\alpha}{R}=: \underline{\lambda},
$$

where $\underline{k}=\inf \operatorname{supp}(\tilde{K})$. We call this latter equation the no bankruptcy condition. Note that

$$
\underline{\lambda}=\bar{\lambda}+\frac{\beta+\underline{k}}{R} .
$$

Therefore, the solvency constraint (16) is a stronger condition on the strategies than the no bankruptcy condition (18), if $\beta>-\underline{k}$, i.e. if higher shocks (greater than $\beta$ ) on the

\footnotetext{
${ }^{5} \operatorname{supp}(\eta)$ denotes the support of $\eta$.
} 
insurance market and small dividends (less the $\bar{\eta}-\beta$ ) in the financial market do not occur simultaneously, which is not a realistic assumption. This is due to the fact that the solvency constraint does not care about dividends, and thus does not take into consideration the (positive) correlation between shocks and dividends, such that higher shocks will have a smaller impact on the wealth evolution since they correspond to higher dividends. If $\beta<-\underline{k}$ (which is the most common case, as for example when insurance shocks and dividends are considered independent), the no bankruptcy condition (18) is stronger than the solvency constraint and thus investors just care about the no bankruptcy condition (18). In this case, the solvency constraint (16) does not eliminate bankruptcy! In the sequel we make the following assumption on the joint distribution of $(\eta, d)$ :

\section{Assumption 6 (Shocks and dividends joint distribution).}

For all $\delta_{1}>0$ and $\delta_{2}>0$,

$$
\mathbb{P}\left[\eta>\bar{\eta}-\delta_{1}, d \leq \delta_{2}\right]>0,
$$

i.e., big shocks and very small dividends have strictly positive probability to jointly occur.

Assumption 6 implies the following Lemma on the distribution of $d-\eta$.

Lemma 2. For all $\delta>0$,

$$
\mathbb{P}[d-\eta \leq-\bar{\eta}+\delta]>0
$$

and thus $\underline{k}=-\bar{\eta}$, i.e. maximal shocks and zero dividends have strictly positive probability to jointly occur.

Proof.

$$
\begin{aligned}
\mathbb{P} & {[d-\eta \leq-\bar{\eta}+\delta]=\mathbb{P}[d \leq \eta-\bar{\eta}+\delta] } \\
& =\int_{0<\delta_{1}<\delta} \mathbb{P}\left[d \leq \eta-\bar{\eta}+\delta \mid \eta-\bar{\eta}>-\delta_{1}\right] d \mathbb{P}\left[\eta-\bar{\eta}>-\delta_{1}\right] \\
& \geq \int_{0<\delta_{1}<\delta} \mathbb{P}\left[d \leq-\delta_{1}+\delta \mid \eta-\bar{\eta}>-\delta_{1}\right] d \mathbb{P}\left[\eta-\bar{\eta}>-\delta_{1}\right] \\
& =\int_{0<\delta_{1}<\delta} \underbrace{\frac{\mathbb{P}\left[d \leq-\delta_{1}+\delta, \eta>\bar{\eta}-\delta_{1}\right]}{\mathbb{P}\left[\eta>\bar{\eta}-\delta_{1}\right]}}_{>0} d \mathbb{P}\left[\eta-\bar{\eta}>-\delta_{1}\right] \\
& >0
\end{aligned}
$$

Thus, $\tilde{K}(-\bar{\eta}+\delta)>0$ for all $\delta>0$, i.e. $\underline{k}=-\bar{\eta}$.

Under Assumption 6, the strategy $\underline{\lambda}$ corresponds to $\frac{R-\bar{\eta}+\beta-\alpha}{R}$ and is a stronger condition on the strategies than the solvency constraint, since obviously $\beta<-\bar{\eta}$. From now on, we take

$$
\underline{\lambda}=\frac{R-\bar{\eta}+\beta-\alpha}{R} .
$$


Let us now consider a single survivor $j$ with a simple strategy $\lambda^{j}>\underline{\lambda}$. Then at each period she will have a strictly positive probability of going bankrupt and therefore $\mathbb{P}\left[j \in \cap_{t} \mathcal{I}_{t}\right]=0$, meaning that she will vanish almost surely from the market. We state these results in the following Lemma.

Lemma 3. Let $\mathcal{I}_{t}=\{j\}$ for some $t$ and $j \in\{1, \ldots, I\}$, i.e. investor $j$ is the unique survivor at time $t$. The following holds:

(i) If $\lambda_{t}^{j}>\underline{\lambda}$, then investor $j$ has strictly positive probability of going bankrupt during period $t+1$.

(ii) If $\lambda_{s}^{j}>\underline{\lambda}$ for all $s \geq t$, then investor $j$ will almost surely eventually vanish from the market. In particular, if investor $j$ uses a simple strategy $\lambda^{j}>\underline{\lambda}$, then she will eventually almost surely vanish from the market almost surely.

Let us now consider the case $\left|\mathcal{I}_{t}\right|>1$. Without loss of generality we set $\mathcal{I}_{t}=\{1,2\}$ : if $\mathcal{I}_{t}=\left\{i_{1}, \ldots, i_{n}\right\}$ with $n=\left|\mathcal{I}_{t}\right|>2$, then we can still reduce the original setting to a 2 -investors setting by defining a "new investor" with strategy $\xi_{s} \in[0,1]$ at time $s \in\{t, t+1\}$ and wealth $w_{s}$, where

$$
\xi_{s}=\frac{\sum_{l=2}^{n} \lambda_{s}^{i_{l}} w_{s}^{i_{l}}}{\sum_{l=2}^{n} w_{s}^{i_{l}}}, \quad w_{s}=\sum_{l=2}^{n} w_{s}^{i_{l}} .
$$

The price of the risky asset at time $s \in\{t, t+1\}$ is then given by $q_{s}=\lambda_{s}^{i_{1}} w_{t}^{i_{1}}+\xi_{s} w_{s}$. Thus let us assume that $\mathcal{I}_{t}=\{1,2\}$. Then from the wealth evolution (17) it follows immediately that for $i=1,2$

$$
i \in \mathcal{I}_{t+1} \Leftrightarrow R\left(1-\lambda_{t}^{i}\right)+d_{t+1} W_{t} \frac{\lambda_{t}^{i}}{q_{t}}+w_{t+1}^{j} \lambda_{t+1}^{j} \frac{\lambda_{t}^{i}}{q_{t}}-\left(\eta_{t+1}-\beta+\alpha\right)>0,
$$

where $j \neq i$.

Proof. (i) Suppose that $i \in \mathcal{I}_{t+1}$. Then $w_{t+1}^{i}>0$ and by equation (17)

$$
\begin{aligned}
w_{t+1}^{i}=R & \left(1-\lambda_{t}^{i}\right) w_{t}^{i}+d_{t+1} W_{t} \frac{\lambda_{t}^{i} w_{t}^{i}}{q_{t}}+ \\
& +\left(w_{t+1}^{1} \lambda_{t+1}^{1}+w_{t+1}^{2} \lambda_{t+1}^{2}\right) \frac{\lambda_{t}^{i} w_{t}^{i}}{q_{t}}-\left(\eta_{t+1}-\beta+\alpha\right) w_{t}^{i},
\end{aligned}
$$

and thus

$$
\begin{aligned}
& w_{t+1}^{i}\left(1-\lambda_{t+1}^{i} \frac{\lambda_{t}^{i} w_{t}^{i}}{q_{t}}\right)=R\left(1-\lambda_{t}^{i}\right) w_{t}^{i}+d_{t+1} W_{t} \frac{\lambda_{t}^{i} w_{t}^{i}}{q_{t}} \\
& +w_{t+1}^{j} \lambda_{t+1}^{j} \frac{\lambda_{t}^{i} w_{t}^{i}}{q_{t}}-\left(\eta_{t+1}-\beta+\alpha\right) w_{t}^{i},
\end{aligned}
$$


where $j \neq i$. Since $\lambda_{t+1}^{i} \neq 1$ (solvency restriction), then $\left(1-\lambda_{t+1}^{i} \frac{\lambda_{t}^{i} w_{t}^{i}}{q_{t}}\right)>0$, and thus from $w_{t+1}^{i}>0$ it follows that

$$
R\left(1-\lambda_{t}^{i}\right) w_{t}^{i}+d_{t+1} W_{t} \frac{\lambda_{t}^{i} w_{t}^{i}}{q_{t}}+w_{t+1}^{j} \lambda_{t+1}^{j} \frac{\lambda_{t}^{i} w_{t}^{i}}{q_{t}}-\left(\eta_{t+1}-\beta+\alpha\right) w_{t}^{i}>0 .
$$

Since $i \in \mathcal{I}_{t}$, then $w_{t}^{i}>0$ and therefore dividing the last inequality by $w_{t}^{i}$ we obtain

$$
R\left(1-\lambda_{t}^{i}\right)+d_{t+1} W_{t} \frac{\lambda_{t}^{i}}{q_{t}}+w_{t+1}^{j} \lambda_{t+1}^{j} \frac{\lambda_{t}^{i}}{q_{t}}-\left(\eta_{t+1}-\beta+\alpha\right)>0 .
$$

(ii) Suppose now that

$$
R\left(1-\lambda_{t}^{i}\right)+d_{t+1} W_{t} \frac{\lambda_{t}^{i}}{q_{t}}+w_{t+1}^{j} \lambda_{t+1}^{j} \frac{\lambda_{t}^{i}}{q_{t}}-\left(\eta_{t+1}-\beta+\alpha\right)>0,
$$

where $j \neq i$. Then for $i \in \mathcal{I}_{t}$,

$$
w_{t}^{i}\left[R\left(1-\lambda_{t}^{i}\right)+d_{t+1} W_{t} \frac{\lambda_{t}^{i}}{q_{t}}+w_{t+1}^{j} \lambda_{t+1}^{j} \frac{\lambda_{t}^{i}}{q_{t}}-\left(\eta_{t+1}-\beta+\alpha\right)\right]>0,
$$

and thus $w_{t+1}^{i}>0$, since

$$
w_{t+1}^{i}=\left[w_{t}^{i} \frac{R\left(1-\lambda_{t}^{i}\right)+d_{t+1} W_{t} \frac{\lambda_{t}^{i}}{q_{t}}+w_{t+1}^{j} \lambda_{t+1}^{j} \frac{\lambda_{t}^{i}}{q_{t}}-\left(\eta_{t+1}-\beta+\alpha\right)}{1-\lambda_{t+1}^{i} \frac{\lambda_{t}^{i} w_{t}^{i}}{q_{t}}}\right]^{+}
$$

and $1-\lambda_{t+1}^{i} \frac{\lambda_{t}^{i} w_{t}^{i}}{q_{t}}>0$ by equation (16).

The necessary and sufficient condition (19) for avoiding bankruptcy for investor $i$ also depends on other investors' wealths and strategies, through the term $w_{t+1}^{j} \lambda_{t+1}^{j} \frac{\lambda_{t}^{i}}{w_{t}^{i}}$. Speculating on other investors' behaviour, investor $i$ could essentially put less wealth on the risk-free asset than allowed under the no bankruptcy condition (18). While this would imply a strictly positive probability of going bankrupt when investor $i$ dominates assets' prices, the no bankruptcy condition is not necessary for avoiding almost surely bankruptcy in the presence of competitors, when they significantly invest in the risky asset. However, the no bankruptcy condition is the minimal condition on investment strategies that almost surely eliminates bankruptcy in the presence of each type of competitor. In fact, an investor who systematically violates the no bankruptcy condition (18), will eventually disappear from the market with probability one, if her opponents are investing all their wealth on the risk-free asset, i.e. an investment strategy that systematically violates the no bankruptcy condition 
is almost surely driven out by the risk-free strategy. Thus the no bankruptcy condition is the minimal condition that ensures that each investor will not go bankrupt with probability 1, regardless from other investors' behaviour. In the sequel, because of the long horizon perspective considered here, and following the approach of Liu, Longstaff, and Pan (2003), we use the no bankruptcy condition to ensure that investors almost surely do not face bankruptcy. In their setting, bankruptcy is penalized with minus infinity utility, so that no optimal strategy will allow final negative wealth with strictly positive probability.

\section{The main results}

From the previous section, it is clear that an investor who uses a strategy that does not almost surely eliminate bankruptcy, will eventually disappear from the market, also if at some point in time she is the unique survivor. Thus, looking at the long-run evolution of investors' wealths, a strategy that does not prevent bankruptcy would not be fit, as it is defined in Blume and Easley (1992). Neither can't be evolutionary stable, as defined in Hens and Schenk-Hoppé (2002a) and Evstigineev, Hens, and Schenk-Hoppé (2003), since it will also disappear almost surely if it dominates asset prices, as shown in Lemma 3. Moreover, as long as dividends and liquidity shocks are not positive correlated, the solvency restriction is not enough to avoid bankruptcy. In fact, under the solvency restriction, an investor faces liquidity shocks with strictly positive probability and if her investment strategy provides small payoff (in particular, dividends are small), then the liquidity shock destroys her wealth. Following the discussion of the previous section, we consider the case where the following assumption holds and we study the long run evolution of investors, who are safe enough on their investment positions to be almost surely able to face liquidity shocks.

\section{Assumption 7 (The no bankruptcy condition).}

For all $i \in \mathcal{I}_{t}$ and all $t \in \mathbb{Z}$,

$$
\lambda_{t}^{i} \in[0, \underline{\lambda}] .
$$

Following Hens and Schenk-Hoppé (2002b), we rewrite the wealth dynamics. We define

$$
B_{t}^{i}=\frac{\lambda_{t}^{i} w_{t}^{i}}{\lambda_{t}^{\prime} \mathbf{w}_{t}}
$$

and

$$
A_{t}^{i}=R\left(1-\lambda_{t}^{i}\right) w_{t}^{i}+B_{t}^{i} d_{t+1} W_{t}-\left(\eta_{t+1}-\beta+\alpha\right) w_{t}^{i} .
$$

By Assumption 7, we have

$$
\mathbf{w}_{t+1}=\mathbf{A}_{t}+\mathbf{B}_{t} \lambda_{t+1}^{\prime} \mathbf{w}_{t+1}
$$


or

$$
\left(I-\mathbf{B}_{t} \boldsymbol{\lambda}_{t+1}^{\prime}\right) \mathbf{w}_{t+1}=\mathbf{A}_{t}
$$

where $\mathbf{A}_{t}=\left(A_{t}^{1}, \ldots, A_{t}^{I}\right)^{\prime}, \mathbf{B}_{t}=\left(B_{t}^{1}, \ldots, B_{t}^{I}\right)^{\prime}$ and $I$ is the identity on $\mathbb{R}^{I}$. Note that for $i \notin \mathcal{I}_{t}, A_{t}^{i}=B_{t}^{i}=0$. The inverse of $I-\mathbf{B}_{t} \boldsymbol{\lambda}_{t+1}^{\prime}$ is given by $I+\left(1-\boldsymbol{\lambda}_{t+1}^{\prime} \mathbf{B}_{t}\right)^{-1} \mathbf{B}_{t} \boldsymbol{\lambda}_{t+1}^{\prime}$, provided that $\boldsymbol{\lambda}_{t+1}^{\prime} \mathbf{B}_{t} \neq 1$ (see Horn and Johnson 1985, Sec. 0.7.4). It can be easily checked that $\boldsymbol{\lambda}_{t+1}^{\prime} \mathbf{B}_{t}<1$ if there exits an investor $i \in \mathcal{I}_{t+1}$ with $\lambda_{t}^{i}<1$ and $\lambda_{t+1}^{i}>0$ and this is still the case when $\left|\mathcal{I}_{t}\right|>1$, by Assumptions 5 and 7 . If $\mathcal{I}_{t}=\{j\}$ for some $j$, then investor $j$ is already the unique survivor and the wealth evolution is easily obtained. Therefore, in the sequel we only consider the case $\left|\mathcal{I}_{t}\right|>1$. Under the assumption of no default during period $t+1$, the wealth evolution can then be written as

$$
\begin{aligned}
\mathbf{w}_{t+1} & =\left(I-\mathbf{B}_{t} \boldsymbol{\lambda}_{t+1}^{\prime}\right)^{-1} \mathbf{A}_{t} \\
& =\left(I+\frac{\mathbf{B}_{t} \boldsymbol{\lambda}_{t+1}^{\prime}}{1-\boldsymbol{\lambda}_{t+1}^{\prime} \mathbf{B}_{t}}\right) \mathbf{A}_{t}
\end{aligned}
$$

and the $i$-th component is given by

$$
\begin{aligned}
w_{t+1}^{i} & =\frac{w_{t}^{i}}{\sum_{j}\left(1-\lambda_{t+1}^{j}\right) \lambda_{t}^{j} w_{t}^{j}} \times \\
& \times\left[d_{t+1} W_{t} \lambda_{t}^{i}+\left[R\left(\underline{\lambda}-\lambda_{t}^{i}\right)+\left(\bar{\eta}-\eta_{t+1}\right)\right]\left(\lambda_{t}^{\prime} \mathbf{w}_{t}+\sum_{j \neq i}\left(\lambda_{t}^{i}-\lambda_{t}^{j}\right) \lambda_{t+1}^{j} w_{t}^{j}\right)\right] .
\end{aligned}
$$

This result is explicitly derived in the Appendix 6.2. We use that

$$
\underline{\lambda}=\frac{R-\bar{\eta}+\beta-\alpha}{R} \Longleftrightarrow R+\beta-\alpha=R \underline{\lambda}+\bar{\eta} .
$$

The price at time $t+1$ follows:

$$
q_{t+1}=\boldsymbol{\lambda}_{t+1}^{\prime} \mathbf{A}_{t}+\frac{\boldsymbol{\lambda}_{t+1}^{\prime} \mathbf{B}_{t} \lambda_{t+1}^{\prime} \mathbf{A}_{t}}{1-\boldsymbol{\lambda}_{t+1}^{\prime} \mathbf{B}_{t}}=\frac{\lambda_{t+1}^{\prime} \mathbf{A}_{t}}{1-\boldsymbol{\lambda}_{t+1}^{\prime} \mathbf{B}_{t}}
$$

Let $r_{t}^{i}=\frac{w_{t}^{i}}{W_{t}}$, and $\zeta_{t}^{i}=\frac{\lambda_{t}^{i}}{\lambda}$ for $i=1, \ldots, I$ and $t \in \mathbb{Z}$. The vector $\mathbf{r}_{t}=\left(r_{t}^{1}, \ldots, r_{t}^{I}\right)^{\prime}$ is the vector of wealth shares, i.e. $\mathbf{r}_{t} \in \Delta^{I-1}=\left\{\mathbf{r} \in \mathbb{R}_{+}^{i} \mid \sum_{i} r_{t}^{i}=1\right\}$. By Assumption $7, \zeta_{t}^{i} \in[0,1]$ and $\zeta_{t}^{i}=1$ iff $\lambda_{t}^{i}=\underline{\lambda}$. We obtain

$$
\begin{aligned}
& w_{t+1}^{i}=\frac{r_{t}^{i} W_{t}}{\sum_{j}\left(1-\underline{\lambda} \zeta_{t+1}^{j}\right) \zeta_{t}^{j} r_{t}^{j}} \times \\
& \quad \times\left[d_{t+1} \zeta_{t}^{i}+\left[R \underline{\lambda}\left(1-\zeta_{t}^{i}\right)+\left(\bar{\eta}-\eta_{t+1}\right)\right]\left(\boldsymbol{\zeta}_{t}^{\prime} \mathbf{r}_{t}+\underline{\lambda} \sum_{j \neq i}\left(\zeta_{t}^{i}-\zeta_{t}^{j}\right) \zeta_{t+1}^{j} r_{t}^{j}\right)\right] .
\end{aligned}
$$


Let $\theta_{t+1}$ be defined by

$$
\begin{aligned}
\theta_{t+1}= & \boldsymbol{\zeta}_{t}^{\prime} \mathbf{r}_{t} d_{t+1}+ \\
& +\sum_{k}\left(R \underline{\lambda}\left(1-\zeta_{t}^{k}\right)+\left(\bar{\eta}-\eta_{t+1}\right)\right) r_{t}^{k}\left(\boldsymbol{\zeta}_{t}^{\prime} \mathbf{r}_{t}+\underline{\lambda} \sum_{j}\left(\zeta_{t}^{k}-\zeta_{t}^{j}\right) \zeta_{t+1}^{j} r_{t}^{j}\right) .
\end{aligned}
$$

Then

$$
W_{t+1}=\frac{\theta_{t+1}}{\sum_{j}\left(1-\underline{\lambda} \zeta_{t+1}^{j}\right) \zeta_{t}^{j} r_{t}^{j}} W_{t} .
$$

The ratio $\frac{\theta_{t+1}}{\sum_{j}\left(1-\underline{\lambda} \zeta_{t+1}^{j}\right) \zeta_{t}^{j} r_{t}^{j}}$ is the growth rate of the economy. From the wealth evolution of equation $(20)$, we obtain the evolution of wealth shares:

$$
\begin{aligned}
r_{t+1}^{i} & =\frac{r_{t}^{i}}{\theta_{t+1}} \times \\
& \times\left[d_{t+1} \zeta_{t}^{i}+\left[R \underline{\lambda}\left(1-\zeta_{t}^{i}\right)+\left(\bar{\eta}-\eta_{t+1}\right)\right]\left(\boldsymbol{\zeta}_{t}^{\prime} \mathbf{r}_{t}+\underline{\lambda} \sum_{j}\left(\zeta_{t}^{i}-\zeta_{t}^{j}\right) \zeta_{t+1}^{j} r_{t}^{j}\right)\right] .
\end{aligned}
$$

From this last equation it follows directly that $r_{t+1}^{i}=0$ if $r_{t}^{i}=0$ and therefore also, $r_{t+1}^{i}=1$ if $r_{t}^{i}=1$.

Without any additional assumption on the dividend process, the liquidity shock factor and the investment strategies we are now able to prove that a trading strategy that corresponds to the no bankruptcy boundary $\underline{\lambda}$, is almost surely driven out by any strategy that is bounded away from $\underline{\lambda}$. From equation (21) it follows that for $i, k \in \mathcal{I}_{t}$,

$$
\begin{aligned}
\frac{r_{t+1}^{i}}{r_{t+1}^{k}} & =\left(\frac{r_{t}^{i}}{r_{t}^{k}}\right) \times \\
& \times \frac{d_{t+1} \zeta_{t}^{i}+\left[R \underline{\lambda}\left(1-\zeta_{t}^{i}\right)+\left(\bar{\eta}-\eta_{t+1}\right)\right]\left(\boldsymbol{\zeta}_{t}^{T} \mathbf{r}_{t}+\underline{\lambda} \sum_{j \neq i}\left(\zeta_{t}^{i}-\zeta_{t}^{j}\right) \zeta_{t+1}^{j} r_{t}^{j}\right)}{d_{t+1} \zeta_{t}^{k}+\left[R \underline{\lambda}\left(1-\zeta_{t}^{k}\right)+\left(\bar{\eta}-\eta_{t+1}\right)\right]\left(\boldsymbol{\zeta}_{t}^{T} \mathbf{r}_{t}+\underline{\lambda} \sum_{j \neq k}\left(\zeta_{t}^{k}-\zeta_{t}^{j}\right) \zeta_{t+1}^{j} r_{t}^{j}\right)} .
\end{aligned}
$$

Let us now suppose that only two investors exist. The first investor is using a simple strategy corresponding to the no bankruptcy boundary, i.e. $\lambda_{t}^{1}=\underline{\lambda}$ for all $t$. The second investor is using a strategy which is bounded away from the no bankruptcy condition, as well as from the strategy putting the wealth only on the risk-free asset, i.e. $\tilde{\delta}<\lambda_{t}^{2}<\underline{\lambda}-\tilde{\delta}$ for all $t>0$ and for some $\tilde{\delta}>0$. Using the notation introduced above, we have $\zeta_{t}^{1}=1$ for all $t$ and $\zeta_{t}^{2} \in(\delta, 1-\delta)$ for all $t$ and $\delta=\frac{\tilde{\delta}}{\underline{\lambda}}>0$. We obtain the following result.

Theorem 1. Under Assumptions 3-7, and given an investor with $\zeta_{t}^{1}=1$ for all $t>0$ and an investor with $\zeta_{t}^{2} \in(\delta, 1-\delta)$ for all $t>0$ and some $\delta>0$, the investor with the simple 
strategy corresponding to the no bankruptcy boundary, will almost surely vanish from the market.

Proof. On $\left\{d_{t+1}-\eta_{t+1}>-\bar{\eta}\right\}$ (by Assumptions 4 and Assumption 6, this set has probability one) we have

$$
\begin{aligned}
& \frac{r_{t+1}^{2}}{r_{t+1}^{1}}=\left(\frac{r_{t}^{2}}{r_{t}^{1}}\right) \frac{d_{t+1} \zeta_{t}^{2}+\left[R \underline{\lambda}\left(1-\zeta_{t}^{2}\right)+\left(\bar{\eta}-\eta_{t+1}\right)\right]\left(\boldsymbol{\zeta}_{t}^{T} \mathbf{r}_{t}-\left(1-\zeta_{t}^{2}\right) \underline{\lambda} r_{t}^{1}\right)}{d_{t+1}+\left(\bar{\eta}-\eta_{t+1}\right)\left(\boldsymbol{\zeta}_{t}^{T} \mathbf{r}_{t}+\left(1-\zeta_{t}^{2}\right) \underline{\lambda} \zeta_{t+1}^{2} r_{t}^{2}\right)} \\
& =\left(\frac{r_{t}^{1}}{r_{t}^{2}}\right) \times \\
& \times \frac{d_{t+1} \zeta_{t}^{2}+\left[R \underline{\lambda}\left(1-\zeta_{t}^{2}\right)+\left(\bar{\eta}-\eta_{t+1}\right)\right]\left[1-\left(1-\zeta_{t}^{2}\right) \underline{\lambda}-\left(1-\zeta_{t}^{2}\right)(1-\underline{\lambda}) r_{t}^{2}\right]}{d_{t+1}+\left(\bar{\eta}-\eta_{t+1}\right)\left[1-\left(1-\zeta_{t}^{2}\right)\left(1-\underline{\lambda} \zeta_{t+1}^{2}\right) r_{t}^{2}\right]} \\
& \geq\left(\frac{r_{t}^{1}}{r_{t}^{2}}\right) \min \left(\frac{d_{t+1} \zeta_{t}^{2}+\left[1-\left(1-\zeta_{t}^{2}\right) \underline{\lambda}\right]\left[R \underline{\lambda}\left(1-\zeta_{t}^{2}\right)+\left(\bar{\eta}-\eta_{t+1}\right)\right]}{d_{t+1}+\left(\bar{\eta}-\eta_{t+1}\right)},\right. \\
& \left.\zeta_{t}^{2} \frac{d_{t+1}+\left[R \underline{\lambda}\left(1-\zeta_{t}^{2}\right)+\left(\bar{\eta}-\eta_{t+1}\right)\right]}{d_{t+1}+\left(\bar{\eta}-\eta_{t+1}\right)\left[\zeta_{t}^{2}\left(1-\underline{\lambda} \zeta_{t+1}^{2}\right)+\underline{\lambda} \zeta_{t+1}^{2}\right]}\right) \\
& \geq\left(\frac{r_{t}^{1}}{r_{t}^{2}}\right) \min \left(\frac{d_{t+1} \zeta_{t}^{2}+\left[1-\left(1-\zeta_{t}^{2}\right) \underline{\lambda}\right]\left[R \underline{\lambda}\left(1-\zeta_{t}^{2}\right)+\left(\bar{\eta}-\eta_{t+1}\right)\right]}{d_{t+1}+\left(\bar{\eta}-\eta_{t+1}\right)},\right. \\
& \left.\zeta_{t}^{2} \frac{d_{t+1}+\left[R \underline{\lambda}\left(1-\zeta_{t}^{2}\right)+\left(\bar{\eta}-\eta_{t+1}\right)\right]}{d_{t+1}+\bar{\eta}-\eta_{t+1}}\right) \\
& \geq\left(\frac{r_{t}^{1}}{r_{t}^{2}}\right) \frac{R \underline{\lambda}(1-\delta) \delta}{d_{t+1}+\bar{\eta}-\eta_{t+1}}>0 \text {. }
\end{aligned}
$$

By the first inequality, we use that

$$
r \mapsto \frac{d \zeta+[R \underline{\lambda}(1-\zeta)+(\bar{\eta}-\eta)][1-(1-\zeta) \underline{\lambda}-(1-\zeta)(1-\underline{\lambda}) r]}{d+(\bar{\eta}-\eta)[1-(1-\zeta)(1-\underline{\lambda} \tilde{\zeta}) r]}
$$

is strictly increasing, strictly decreasing or flat as a function of $r$, depending on the parameters $d, \eta, \zeta, \tilde{\zeta}, \underline{\lambda}$ and $R$. Thus, the minimum of the function is attained for $r=1$ or $r=0$. By the second inequality, we use that

$$
\left[\zeta_{t}^{2}\left(1-\underline{\lambda} \zeta_{t+1}^{2}\right)+\underline{\lambda} \zeta_{t+1}^{2}\right]<1
$$

for all $\zeta_{t}^{2}, \zeta_{t+1}^{2} \in[0,1]$.

Iteratively, we obtain

$$
\log \frac{r_{t+1}^{2}}{r_{t+1}^{1}} \geq \sum_{\tau=1}^{t+1} \log \left(\frac{R \underline{\lambda}(1-\delta) \delta}{d_{\tau}+\bar{\eta}-\eta_{\tau}}\right)+\log \frac{r_{0}^{2}}{r_{0}^{1}}
$$


Let $\epsilon<R(1-\delta) \delta \underline{\lambda}$, then

$$
\log \frac{r_{t+1}^{2}}{r_{t+1}^{1}} \geq C \sum_{\tau=1}^{t+1} 1_{\left\{d_{\tau}+\bar{\eta}-\eta_{\tau} \leq \epsilon\right\}}+\log \frac{r_{0}^{2}}{r_{0}^{1}}
$$

where $C=\log \frac{R(1-\delta) \delta \underline{\underline{\lambda}}}{\epsilon}>0$ by definition of $\epsilon$. By the Theorem,

$$
\begin{aligned}
\lim _{t \rightarrow \infty} \frac{1}{t+1} \log \frac{r_{t+1}^{2}}{r_{t+1}^{1}} & \geq C \lim _{t \rightarrow \infty} \frac{1}{t+1}\left(\sum_{\tau=1}^{t+1} 1_{\left\{d_{\tau}+\bar{\eta}-\eta_{\tau} \leq \epsilon\right\}}+\log \frac{r_{0}^{2}}{r_{0}^{1}}\right) \\
& =C \tilde{K}(d-\eta \leq-\bar{\eta}+\epsilon)=\gamma>0,
\end{aligned}
$$

by Assumption 6. Thus $\frac{r_{t}^{2}}{1-r_{t}^{2}}=\frac{r_{t}^{2}}{r_{t}^{1}} \approx \exp (t \gamma) \rightarrow \infty$ as $t \rightarrow \infty$, i.e $r_{t}^{2} \rightarrow 1$ almost surely.

The theorem states that, while being at the boundary of the no-bankruptcy condition means that bankruptcy is excluded with probability one, the market selection mechanism still forces such an investor to vanish from the market, if other investors are using strategies that are bounded away from $\underline{\lambda}$. Therefore, the trading strategy $\lambda_{t}^{i}=\underline{\lambda}$ cannot be evolutionary stable as defined by Evstigineev, Hens, and Schenk-Hoppé (2003). In fact, even if this strategy possesses almost the entire wealth, an investment strategy that is bounded away from $\underline{\lambda}$ is able to drastically perturb the distribution of wealth shares and to drive out $\lambda_{t}^{i}$. We next ask the question about investment strategies that are evolutionary stable, referring to Hens and Schenk-Hoppé (2002a) and Evstigineev, Hens, and Schenk-Hoppé (2003). The evolution of wealth shares from equation (21) can be written as follows. For $i=1, \ldots, I$ let

$$
\begin{aligned}
f^{i}\left(\mathbf{r}_{t}, t\right)=\frac{r_{t}^{i}}{\theta_{t+1}}\left[d_{t+1} \zeta_{t}^{i}+(R \underline{\lambda}\right. & \left.\left(1-\zeta_{t}^{i}\right)+\left(\bar{\eta}-\eta_{t+1}\right)\right) \times \\
& \left.\times\left(\boldsymbol{\zeta}_{t}^{\prime} \mathbf{r}_{t}+\underline{\lambda} \sum_{j}\left(\zeta_{t}^{i}-\zeta_{t}^{j}\right) \zeta_{t+1}^{j} r_{t}^{j}\right)\right] .
\end{aligned}
$$

Then

$$
r_{t+1}^{i}=f^{i}\left(\mathbf{r}_{t}, t\right)
$$

or

$$
\mathbf{r}_{t+1}=f\left(\mathbf{r}_{t}, t\right)
$$

where $f=\left(f^{1}, \ldots, f^{I}\right)^{\prime}$. Although it does not appear explicitly in the definition of $f_{t}^{i}$, the function $f_{t}$ also depends on the state of the world $s^{t+1}$ up to time $t+1$, through investors' strategies at time $t+1$, the dividend $d_{t+1}$ and the liquidity shock factor $\eta_{t+1}$. We make the following additional assumption on the trading strategies to make $f$ independent from $t$ and, therefore, the market selection mechanism stationary, also because Assumptions 3 and 4 on $\left(\eta_{t}\right)_{t \in \mathbb{Z}}$ and $\left(d_{t}\right)_{t \in \mathbb{Z}}$, respectively. 


\section{Assumption 8 (Stationary trading strategies).}

The trading strategies are stationary, i.e. for all $t \in \mathbb{Z}$ and all $i \in \mathcal{I}_{t}$

$$
\lambda_{t}^{i}\left(s^{t}\right)=\lambda^{i}\left(s^{t}\right)
$$

The market selection process (23) generates a random dynamical system (see Arnold 1998) on the simplex $\Delta^{I-1}$. Given a vector of initial wealth shares $\mathbf{r} \in \Delta^{I-1}$ and $t>0$, the map

$$
\phi(t, \omega, \mathbf{r})=f\left(s^{t}, \cdot\right) \circ f\left(s^{t-1}, \cdot\right) \circ \cdots \circ f\left(s^{1}, \mathbf{r}\right),
$$

on $\mathbb{N} \times \Omega \times \Delta^{I-1}$ gives the investors' wealth shares at time $t$, if the state of the world is $\omega=\left(s_{t}\right)_{t \in \mathbb{Z}}$, and $\phi(0, \omega, \mathbf{r})=\mathbf{r}$. In the sequel we characterize vectors of wealth shares that are invariant under $\phi$. We introduce the following definition.

Definition 1 (Fixed point). The vector of relative wealth shares $\mathbf{r} \in \Delta^{I-1}$ is called a deterministic fixed point of $\phi$, if and only if

$$
\phi(t, \mathbf{r}, \cdot)=\mathbf{r}
$$

almost surely for all $t$. The distribution of market shares $\mathbf{r}$ is said to be invariant under the market selection process (23).

Clearly, $\mathbf{r}$ is a deterministic fixed point of $\phi$ if and only if $f(\mathbf{r})=\phi(1, \mathbf{r}, \cdot)=\mathbf{r}$ almost surely. Therefore, the vectors of wealth shares $\mathbf{r}=\mathbf{e}^{i}$ for $i=1, \ldots, I$ are deterministic fixed points of $\phi$, where

$$
e^{i, j}=\left\{\begin{array}{ll}
1 & \text { if } j=i \\
0 & \text { else }
\end{array} .\right.
$$

The following lemma shows that $\mathbf{e}^{i}$ are the unique deterministic fixed points of $\phi$. This result also holds if Assumption 8 is not satisfied.

Lemma 4. Let $\mathbf{r}$ be a deterministic fixed point of $\phi$. Then $\mathbf{r}=\mathbf{e}^{i}$ for some $i=1, \ldots, I$.

Proof. Let assume that $r^{i}=r_{t+1}^{i}=r_{t}^{i} \in(0,1)$. Then

$$
\theta_{t+1}=d_{t+1} \zeta_{t}^{i}+\left(R \underline{\lambda}\left(1-\zeta_{t}^{i}\right)+\left(\bar{\eta}-\eta_{t+1}\right)\right)\left(\zeta_{t}^{\prime} \mathbf{r}_{t}+\underline{\lambda} \sum_{j}\left(\zeta_{t}^{i}-\zeta_{t}^{j}\right) \zeta_{t+1}^{j} r_{t}^{j}\right),
$$

or equivalently

$$
\begin{aligned}
& d_{t+1} \sum_{k \neq i} \zeta_{t}^{k} r_{t}^{k}+ \\
& +\sum_{k \neq i}\left(R \underline{\lambda}\left(1-\zeta_{t}^{k}\right)+\left(\bar{\eta}-\eta_{t+1}\right)\right) r_{t}^{k}\left(\boldsymbol{\zeta}_{t}^{\prime} \mathbf{r}_{t}+\underline{\lambda} \sum_{j}\left(\zeta_{t}^{k}-\zeta_{t}^{j}\right) \zeta_{t+1}^{j} r_{t}^{j}\right) \\
= & d_{t+1} \zeta_{t}^{i}\left(1-r_{t}^{i}\right)+ \\
& +\left(1-r_{t}^{i}\right)\left(R \underline{\lambda}\left(1-\zeta_{t}^{i}\right)+\left(\bar{\eta}-\eta_{t+1}\right)\right)\left(\boldsymbol{\zeta}_{t}^{\prime} \mathbf{r}_{t}+\underline{\lambda} \sum_{j}\left(\zeta_{t}^{i}-\zeta_{t}^{j}\right) \zeta_{t+1}^{j} r_{t}^{j}\right) .
\end{aligned}
$$


Since $1-r_{t}^{i}=\sum_{k \neq i} r_{t}^{k}$, the right-hand side of equation (25) corresponds to

$$
d_{t+1} \sum_{k \neq i} \zeta_{t}^{i} r_{t}^{k}+\sum_{k \neq i}\left(R \underline{\lambda}\left(1-\zeta_{t}^{i}\right)+\left(\bar{\eta}-\eta_{t+1}\right)\right) r_{t}^{k}\left(\boldsymbol{\zeta}_{t}^{\prime} \mathbf{r}_{t}+\underline{\lambda} \sum_{j}\left(\zeta_{t}^{i}-\zeta_{t}^{j}\right) \zeta_{t+1}^{j} r_{t}^{j}\right)
$$

and thus equation (25) is equivalent to

$$
\begin{aligned}
0= & d_{t+1} \sum_{k \neq i}\left(\zeta_{t}^{i}-\zeta_{t}^{k}\right) r_{t}^{k}+\zeta_{t}^{\prime} \mathbf{r}_{t} R \underline{\lambda} \sum_{k \neq i}\left(\zeta_{t}^{k}-\zeta_{t}^{i}\right) r_{t}^{k} \\
& +\sum_{k \neq i}\left(R \underline{\lambda}\left(1-\zeta_{t}^{i}\right)+\left(\bar{\eta}-\eta_{t+1}\right)\right) r_{t}^{k} \underline{\lambda} \sum_{j}\left(\zeta_{t}^{i}-\zeta_{t}^{j}\right) \zeta_{t+1}^{j} r_{t}^{j} \\
& +\sum_{k \neq i}\left(R \underline{\lambda}\left(1-\zeta_{t}^{k}\right)+\left(\bar{\eta}-\eta_{t+1}\right)\right) r_{t}^{k} \underline{\lambda} \sum_{j}\left(\zeta_{t}^{j}-\zeta_{t}^{k}\right) \zeta_{t+1}^{j} r_{t}^{j} \\
= & d_{t+1} \sum_{k}\left(\zeta_{t}^{i}-\zeta_{t}^{k}\right) r_{t}^{k}+\underline{\lambda}\left(\bar{\eta}-\eta_{t+1}\right) \sum_{k}\left(\zeta_{t}^{i}-\zeta_{t}^{k}\right) r_{t}^{k} \sum_{j} \zeta_{t+1}^{j} r_{t}^{j} \\
& +R \underline{\lambda}^{2} \sum_{k}\left(\zeta_{t}^{i}-\zeta_{t}^{k}\right) r_{t}^{k} \sum_{j}\left(1+\zeta_{t}^{j}\right) \zeta_{t+1}^{j} r_{t}^{j} \\
& -R \underline{\lambda}^{2} \sum_{k}\left(\zeta_{t}^{i}-\zeta_{t}^{k}\right)\left(\zeta_{t}^{i}+\zeta_{t}^{k}\right) r_{t}^{k} \sum_{j} \zeta_{t+1}^{j} r_{t}^{j}-R \underline{\lambda} \sum_{k}\left(\zeta_{t}^{i}-\zeta_{t}^{k}\right) r_{t}^{k} \sum_{j} \zeta_{t}^{j} r_{t}^{j} .
\end{aligned}
$$

Let us first suppose that $\sum_{k}\left(\zeta_{t}^{i}-\zeta_{t}^{k}\right) r_{t}^{k}=0$. Then $\zeta_{i}=\xi_{t}$, where $\xi_{t}=\frac{\sum_{k \neq i} \zeta_{t}^{k} r_{t}^{k}}{1-r_{t}^{i}}$. Moreover, the last equation is equivalent to

$$
\sum_{k}\left(\zeta_{t}^{i}-\zeta_{t}^{k}\right) \zeta_{t}^{k} r_{t}^{k}=0
$$

and thus

$$
\xi_{t}^{2}=\sum_{k \neq i}\left(\zeta_{t}^{k}\right)^{2} r_{t}^{k}
$$

This last equation implies $\zeta_{t}^{k}=0$ for all $k$, or $r_{t}^{k}=0$ for $k \neq i$. In the first case we have a contradiction to Assumption 5. In the second case we have a contradiction to $r_{t}^{i} \in(0,1)$. Let us now suppose that $\sum_{k}\left(\zeta_{t}^{i}-\zeta_{t}^{k}\right) r_{t}^{k} \neq 0$. Without loss of generality, we take $\sum_{k}\left(\zeta_{t}^{i}-\right.$ 
$\left.\zeta_{t}^{k}\right) r_{t}^{k}>0$ (the same argument can also be used for the case $\sum_{k}\left(\zeta_{t}^{i}-\zeta_{t}^{k}\right) r_{t}^{k}<0$ ). Then

$$
\begin{aligned}
0= & d_{t+1}+\underline{\lambda}\left(\bar{\eta}-\eta_{t+1}\right) \sum_{j} \zeta_{t+1}^{j} r_{t}^{j}-R \underline{\lambda} \sum_{j}\left(1-\underline{\lambda} \zeta_{t+1}^{j}\right) \zeta_{t}^{j} r_{t}^{j} \\
& -\frac{R \underline{\lambda}^{2}}{\sum_{l}\left(\zeta_{t}^{i}-\zeta_{t}^{l}\right) r_{t}^{l}} \sum_{k}\left(\left(\zeta_{t}^{i}\right)^{2}-\left(\zeta_{t}^{k}\right)^{2}-\sum_{l}\left(\zeta_{t}^{i}-\zeta_{t}^{l}\right) r_{t}^{l}\right) r_{t}^{k} \sum_{j} \zeta_{t+1}^{j} r_{t}^{j} \\
= & d_{t+1}+\underline{\lambda}\left(\bar{\eta}-\eta_{t+1}\right) \sum_{j} \zeta_{t+1}^{j} r_{t}^{j}-R \underline{\lambda} \sum_{j}\left(1-\underline{\lambda} \zeta_{t+1}^{j}\right) \zeta_{t}^{j} r_{t}^{j} \\
& -\frac{R \underline{\lambda}^{2}}{\sum_{l}\left(\zeta_{t}^{i}-\zeta_{t}^{l}\right) r_{t}^{l}} \sum_{k \neq i} \zeta_{t}^{k}\left(1-\zeta_{t}^{k}\right) r_{t}^{k} \sum_{j} \zeta_{t+1}^{j} r_{t}^{j} .
\end{aligned}
$$

Since $r_{t+1}^{i}=r_{t}^{i} \in(0,1)$, the set $\left\{\sum_{j} \zeta_{t+1}^{j} r_{t}^{j}=0\right\}$ has probability zero by Assumption 5 . Thus $\sum_{j} \zeta_{t+1}^{j} r_{t}^{j}>0$ almost surely. Let $\delta>0$, then by Assumptions 4 and 6 , the set $\left\{s_{t+1} \mid d_{t+1}\left(s_{t+1}\right)=0, \bar{\eta}-\eta_{t+1}\left(s_{t+1}\right)<\delta\right\}$ has strictly positive probability independently from $s^{t}$. Thus

$$
(1-\underline{\lambda}) \sum_{j} \zeta_{t}^{j} r_{t}^{j}+\frac{\underline{\lambda}}{\sum_{l}\left(\zeta_{t}^{i}-\zeta_{t}^{l}\right) r_{t}^{l}} \sum_{k \neq i} \zeta_{t}^{k}\left(1-\zeta_{t}^{k}\right) r_{t}^{k}<\delta
$$

Since this is true for all $\delta>0, \zeta_{t}^{j}=0$ for all investors with strictly positive wealth share at time $t$, a contradiction to Assumption 5 or $r_{t}^{i}=r_{t+1}^{i} \in(0,1)$. Therefore, $r_{t}^{i}=0$ or $r_{t}^{i}=1$.

The Lemma implies that we can restrict ourselves to monomorphic populations of investors (all investors with a strictly positive market share possess the same trading strategy), to analyse invariant wealth share distributions. In particular, we are looking at deterministic fixed points that are stable, such that a small perturbation of the vector of wealth shares does not change the long-run evolution. Since invariant wealth share distributions correspond to monomorphic populations, the stability of investment strategies is related to the stability of the associated fixed point. Therefore, we consider a population of trading strategies with an incumbent strategy $\lambda^{i}$ (with market share $r_{t}^{i}$ ) and a distinct mutant strategy $\lambda^{j}$ (with market share $\left.r_{t}^{j}=1-r_{t}^{i}\right)$.

Definition 2 (Evolutionary stable strategies). A trading strategy $\lambda^{i}$ is called evolutionary stable if, for all strategies $\lambda^{j}$, there is a random variable $\epsilon>0$ such that $\lim _{t \rightarrow \infty} \phi^{i}(t, \omega, r)=$ 1 for all $r^{i} \geq 1-\epsilon(\omega)$.

If the choice of mutant strategies is restricted to those trading strategies that are a local mutation of $\lambda^{i}$, i.e. there exists a random variable $\delta(\omega)>0$ with $\left|\lambda^{i}(\omega)-\lambda^{j}(\omega)\right|<\delta(\omega)$ almost surely, then $\lambda^{i}$ is called locally evolutionary stable. 
Let us consider a population of only two investors, where the incumbent is investor 1 with market share $r_{t}^{1}$ and the mutant is investor 2 with market share $1-r_{t}^{1}$. The wealth share dynamic for investor 1 is obtained from (21) by

$$
\psi\left(r_{t}^{1}\right)=f^{1}\left(r_{t}^{1}, 1-r_{t}^{1}\right)
$$

The derivative of $\psi$ evaluated at $r_{t}^{1}=1$ corresponds to

$$
\left.\frac{\partial \psi\left(r_{t}^{1}\right)}{\partial r_{t}^{1}}\right|_{r_{t}^{1}=1}=\frac{1}{\zeta_{t}^{1}} \frac{\zeta_{t}^{2} d_{t+1}+\left[R \underline{\lambda}\left(1-\zeta_{t}^{2}\right)+\left(\bar{\eta}-\eta_{t+1}\right)\right]\left[\zeta_{t}^{1}+\left(\zeta_{t}^{2}-\zeta_{t}^{1}\right) \underline{\lambda} \zeta_{t+1}^{1}\right]}{d_{t+1}+R \underline{\lambda}\left(1-\zeta_{t}^{1}\right)+\bar{\eta}-\eta_{t+1}} .
$$

The right-hand side of this last equation corresponds to the exponential growth rate of the trading strategy $\lambda^{2}$, when investor 1 owns total market wealth (see equation (21)). Note that by Theorem 1 , we can impose without loss of generality that $\zeta^{1} \neq 1$. Thus, the derivative

$$
\left.\frac{\partial \psi\left(r_{t}^{1}\right)}{\partial r_{t}^{1}}\right|_{r_{t}^{1}=1}
$$

is well defined and bounded for $\zeta^{2} \in[0,1]$.

We restrict ourselves to the case where the processes $\left(S_{t}\right)_{t \in \mathbb{Z}}$ determining the state of nature is i.i.d. and we denote by $\mu$ the distribution of $S_{t}$ on $S$. Then, the growth rate of investor's 2 market share in a small neighborhood of $r_{t}^{1}=1$ is equal to

$$
g_{\zeta^{1}}\left(\zeta^{2}\right)=\int_{\mathcal{S}^{\mathbb{N}}} \tilde{g}_{\zeta^{1}}\left(\zeta^{2}\left(s^{0}\right), s^{0}\right) \mu^{\mathbb{N}}\left(d s^{0}\right)
$$

where

$$
\begin{aligned}
& \tilde{g}_{\zeta^{1}}\left(\zeta^{2}\left(s^{0}\right), s^{0}\right)= \\
& =\int_{S} \mu(d s) \log \left\{\zeta ^ { 1 } ( s ^ { 0 } ) ^ { - 1 } [ d ( s ) + R \underline { \lambda } ( 1 - \zeta ^ { 1 } ( s ^ { 0 } ) ) + \overline { \eta } - \eta ( s ) ] ^ { - 1 } \left[\zeta^{2}\left(s^{0}\right) d(s)+\right.\right. \\
& \left.\left.\quad+\left(R \underline{\lambda}\left(1-\zeta^{2}\left(s^{0}\right)\right)+(\bar{\eta}-\eta(s))\right)\left(\zeta^{1}\left(s^{0}\right)+\left(\zeta^{2}\left(s^{0}\right)-\zeta^{1}\left(s^{0}\right)\right) \underline{\lambda} \zeta^{1}\left(s^{0}, s\right)\right)\right]\right\} .
\end{aligned}
$$

The function $\zeta^{2}\left(s^{0}\right) \mapsto \tilde{g}_{\zeta^{1}}\left(\zeta^{2}\left(s^{0}\right), s^{0}\right)$ is continuous, strictly concave on $[0,1]$ for all $s^{0}$ and, obviously $\tilde{g}_{\zeta^{1}}\left(\zeta^{1}\left(s^{0}\right), s^{0}\right)=0$ for all $s^{0}$. Moreover,

$$
\begin{aligned}
& \left.\frac{\partial \tilde{g}_{\zeta^{1}}\left(\zeta^{2}\left(s^{0}\right), s^{0}\right)}{\partial \zeta^{2}\left(s^{0}\right)}\right|_{\zeta^{2}\left(s^{0}\right)=\zeta^{1}\left(s^{0}\right)}=\frac{1}{\zeta^{1}\left(s^{0}\right)} \times \\
& \quad \times \int_{S} \mu(d s) \frac{d(s)-R \underline{\lambda} \zeta^{1}\left(s^{0}\right)+R \underline{\lambda}^{2} \zeta^{1}\left(s^{0}, s\right)\left(1-\zeta^{1}\left(s^{0}\right)\right)+(\bar{\eta}-\eta(s)) \underline{\lambda} \zeta^{1}\left(s^{0}, s\right)}{d(s)+\left(R \underline{\lambda}\left(1-\zeta^{1}\left(s^{0}\right)\right)+(\bar{\eta}-\eta(s))\right)} .
\end{aligned}
$$

The following Theorem holds: 
Theorem 2. Let the state of nature $\left(s_{t}\right)_{t \in \mathbb{Z}}$ be determined by an i.i.d. process. Suppose that all investors use simple strategies $\lambda^{i}(\omega) \equiv \lambda^{i} \in(0,1)$.

(i) If

$$
\mathbb{E}\left[\frac{d}{d+\bar{\eta}-\eta}\right]+\underline{\lambda} \mathbb{E}\left[\frac{\bar{\eta}-\eta}{d+\bar{\eta}-\eta}\right] \geq R \underline{\lambda} \mathbb{E}\left[\frac{1}{d+\bar{\eta}-\eta}\right]
$$

then there is no evolutionary stable investment strategy.

(ii) If

$$
\mathbb{E}\left[\frac{d}{d+\bar{\eta}-\eta}\right]+\underline{\lambda} \mathbb{E}\left[\frac{\bar{\eta}-\eta}{d+\bar{\eta}-\eta}\right]<R \underline{\lambda} \mathbb{E}\left[\frac{1}{d+\bar{\eta}-\eta}\right]
$$

then there is a unique evolutionary stable investment strategy $\lambda^{\star}=\underline{\lambda} \zeta^{\star}$ where $\zeta^{\star}$ satisfies:

$$
\int_{S} \mu(d s) \frac{\left[d(s)-R \underline{\lambda} \zeta+R \underline{\lambda}^{2} \zeta(1-\zeta)+(\bar{\eta}-\eta(s)) \underline{\lambda} \zeta\right]}{d(s)+R \underline{\lambda}(1-\zeta)+\bar{\eta}-\eta(s)}=0 .
$$

Proof. An investment strategy $\zeta^{\star}$ is evolutionary stable if $g_{\zeta^{\star}}(\zeta)<0$ for all $\zeta \in[0,1]$. From Theorem 1, we have $\zeta^{\star} \neq 1$. Moreover, if investors strategies are simple, we have

$$
g_{\zeta^{1}}\left(\zeta^{2}\right)=\tilde{g}_{\zeta^{1}}\left(\zeta^{2}\right)
$$

Thus $\zeta^{2} \mapsto g_{\zeta^{1}}\left(\zeta^{2}\right)$ is continuous, strictly concave and $g_{\zeta^{1}}\left(\zeta^{1}\right)=0$. Therefore, $g_{\zeta^{1}}(\zeta)<0$ for all $\zeta \in[0,1]$ if and only if

$$
\left.\frac{d g_{\zeta_{1}}\left(\zeta_{2}\right)}{d \zeta_{2}}\right|_{\zeta_{2}=\zeta_{1}}=0
$$

Hence, $\zeta^{\star}$ is an evolutionary stable investment strategy if it solves

$$
0=\int_{S} \mu(d s) \frac{\left[d(s)-R \underline{\lambda} \zeta+R \underline{\lambda}^{2} \zeta(1-\zeta)+(\bar{\eta}-\eta(s)) \underline{\lambda} \zeta\right]}{d(s)+R \underline{\lambda}(1-\zeta)+\bar{\eta}-\eta(s)} .
$$

Let

$$
h(\zeta ; d, \eta)=\frac{\left[d-R \underline{\lambda} \zeta+R \underline{\lambda}^{2} \zeta(1-\zeta)+(\bar{\eta}-\eta) \underline{\lambda} \zeta\right]}{d+R \underline{\lambda}(1-\zeta)+\bar{\eta}-\eta} .
$$

Then $\frac{\partial h(\zeta ; d, \eta)}{\partial \zeta}<0$ for $\zeta \in[0,1]$, thus $h$ is strictly decreasing on $[0,1]$ for all $d$ and $\eta$ and, $h(0 ; d, \eta)=\frac{d}{d+R \lambda(\bar{\eta}-\eta)} \geq 0$ for all $d$ and $\eta$, and strictly positive for $d>0$. Thus

$$
\int_{\mathcal{S}} \mu(d s) h(0 ; d(s), \eta(s))>0
$$


since by Assumption 4 the set $\{s \in S \mid d(s)>0\}$ has strictly positive probability. Moreover, $h(1 ; d, \eta)=\frac{d-R \underline{\lambda}+(\bar{\eta}-\eta) \underline{\lambda}}{d+\bar{\eta}-\eta}$ and

$$
\begin{gathered}
\int_{S} \mu(d s) h(1 ; d(s), \eta(s))=\int_{S} \mu(d s) \frac{d(s)-R \underline{\lambda}+(\bar{\eta}-\eta(s)) \underline{\lambda}}{d(s)+\bar{\eta}-\eta(s)} \\
=\int_{S} \mu(d s) \frac{d(s)}{d(s)+\bar{\eta}-\eta(s)}+\underline{\lambda} \int_{S} \mu(d s) \frac{\bar{\eta}-\eta(s)}{d(s)+\bar{\eta}-\eta(s)} \\
\quad-R \underline{\lambda} \int_{S} \frac{\mu(d s)}{d(s)+\bar{\eta}-\eta(s)} \\
=\mathbb{E}\left[\frac{d}{d+\bar{\eta}-\eta}\right]+\underline{\lambda} \mathbb{E}\left[\frac{\bar{\eta}-\eta}{d+\bar{\eta}-\eta}\right]-R \underline{\lambda} \mathbb{E}\left[\frac{1}{d+\bar{\eta}-\eta}\right] .
\end{gathered}
$$

Therefore

$$
\int_{\mathcal{S}} \mu(d s) h(1 ; d(s), \eta(s))<0
$$

if and only if

$$
\mathbb{E}\left[\frac{d}{d+\bar{\eta}-\eta}\right]+\underline{\lambda} \mathbb{E}\left[\frac{\bar{\eta}-\eta}{d+\bar{\eta}-\eta}\right]-R \underline{\lambda} \mathbb{E}\left[\frac{1}{d+\bar{\eta}-\eta}\right]<0 .
$$

Thus, if the condition $\mathbb{E}\left[\frac{d}{d+\bar{\eta}-\eta}\right]+\underline{\lambda} \mathbb{E}\left[\frac{\bar{\eta}-\eta}{d+\bar{\eta}-\eta}\right]-R \underline{\lambda} \mathbb{E}\left[\frac{1}{d+\bar{\eta}-\eta}\right] \geq 0$ holds, then the function $\int_{S} \mu(d s) h(\zeta ; d(s), \eta(s))$ is strictly positive on $[0,1)$ and therefore no evolutionary stable strategy exists. Note that since $g_{\zeta^{1}}$ is continuous and strictly concave, if no evolutionary stable strategy exists, then no local evolutionary stable strategy can exist either. In fact, in a small neighborhood of $\zeta_{1}$ there exists an investment strategy $\zeta^{2}>\zeta^{1}$ with $g_{\zeta^{1}}\left(\zeta^{2}\right)>0$. This proves (i).

If $\mathbb{E}\left[\frac{d}{d+\bar{\eta}-\eta}\right]+\underline{\lambda} \mathbb{E}\left[\frac{\bar{\eta}-\eta}{d+\bar{\eta}-\eta}\right]-R \underline{\lambda} \mathbb{E}\left[\frac{1}{d+\bar{\eta}-\eta}\right]<0$, then there exists exactly one $\zeta^{\star} \in(0,1)$ such that $\int_{S} \mu(d s) h\left(\zeta^{\star} ; d(s), \eta(s)\right)=0$ and therefore $g_{\zeta^{\star}}(\zeta)<0$ for $\zeta \in(0,1), \zeta \neq \zeta^{\star}$. Obviously $\zeta^{\star}$ solves

$$
\int_{S} \mu(d s) h(\zeta ; d(s), \eta(s))=0
$$

which proves (ii).

\section{Remark}

From Section 3, simple trading strategies $\lambda>\underline{\lambda}$ disappears almost surely owing to liquidity shocks. Moreover, from Theorem 1, the simple investment strategy at the boundary of the no bankruptcy condition is driven out of the market by any strategy that is bounded away from the no bankruptcy condition and the risk-free asset. The condition for the existence of evolutionary stable strategies given in Theorem 2, relates to the growth rate of a mutant investment strategy in a neighborhood of $\zeta=1$, i.e. $\lambda=\underline{\lambda}$. If this growth rate is strictly positive, then an investor who puts a greater proportion of her wealth into the risky asset than $\zeta=1$, is able to gain wealth share when asset prices are dominated by $\underline{\lambda}$. However, 
this kind of strategy only delivers growth as long as it doesn't lead to bankruptcy, which will almost surely occur. Thus, if the growth rate at $\zeta=1$ is positive, no evolutionary stable strategy can exist.

\section{Example}

Let us suppose that $d$ and $\eta$ are independent, $d$ is distributed on $[0,0.1]$ with $H(0)=\mathbb{P}[d=$ $0]=0.01,(d \mid d>0) \sim \operatorname{Beta}(2,2)$ and, $\eta \sim \operatorname{Uniform}(0.05,0.1)$. Moreover, we take $\alpha=0.04$ and $R=1.025$. Then $\underline{\lambda}=0.93796$ and $\zeta^{\star}=0.213528$. Thus, the unique evolutionary stable investment strategy consists in investing only the $20.03 \%$ of the wealth on the risky asset. The evolution of market shares is shown Figure 1.

If $(d \mid d>0) \sim \operatorname{Beta}(2,3)$, then the unique evolutionary stable strategy is $\lambda^{\star}=17.66 \%$; $\zeta^{\star}$ decreases with decreasing $\mathbb{E}[d]$.

\section{Conclusion}

In this paper we have proposed an evolutionary portfolio model with bankruptcy. The investors are insurances companies, thus with insurance market exposures. The amount of wealth that is withdrawn or collected at each period, corresponds to the difference between indemnities, which must be paid out and premia paid in. If this difference is negative, insurance companies face liquidity shocks that could force investors to withdraw their entire wealth, thus forcing the company into bankruptcy. We introduce the no bankruptcy condition on investment strategies that ensures that bankruptcy is excluded with probability one and we analyse the set of simple strategies that are evolutionary stable if the state of nature is generated by an i.i.d. process. In fact, if an investment strategy does not almost surely eliminate bankruptcy, the company will almost surely disappear from the market eventually. We prove that invariant wealth shares distribution only corresponds to monomorphic populations. Moreover, we show that, depending on the dividend and liquidity shock processes, there exists a unique evolutionary stable strategy or, for all strategies there exists at least one mutant that is able to drastically perturb the distribution of wealth shares. We give the condition that characterize existence and, if this condition is satisfied we also characterize the unique evolutionary stable strategy.

This work shows that when savings and withdrawals are not a positive percentage of the investor's wealth, then one should also take bankruptcy into account. Thus, an additional dimension has to be considered as compared to the case where bankruptcy is excluded almost surely (Blume and Easley 2002, Evstigineev, Hens, and Schenk-Hoppé 2003). Here we take a long-horizon perspective, so that a trading strategy with strictly positive probability of going bankrupt at each period cannot be evolutionary fit, since it will disappear from the market almost surely. This paper also suggest that near the growth rate, one should also take the risk dimension into account, which is the probability of going bankrupt. Thus we introduce the risk dimension in the Evolutionary Portfolio Theory. 
(a)

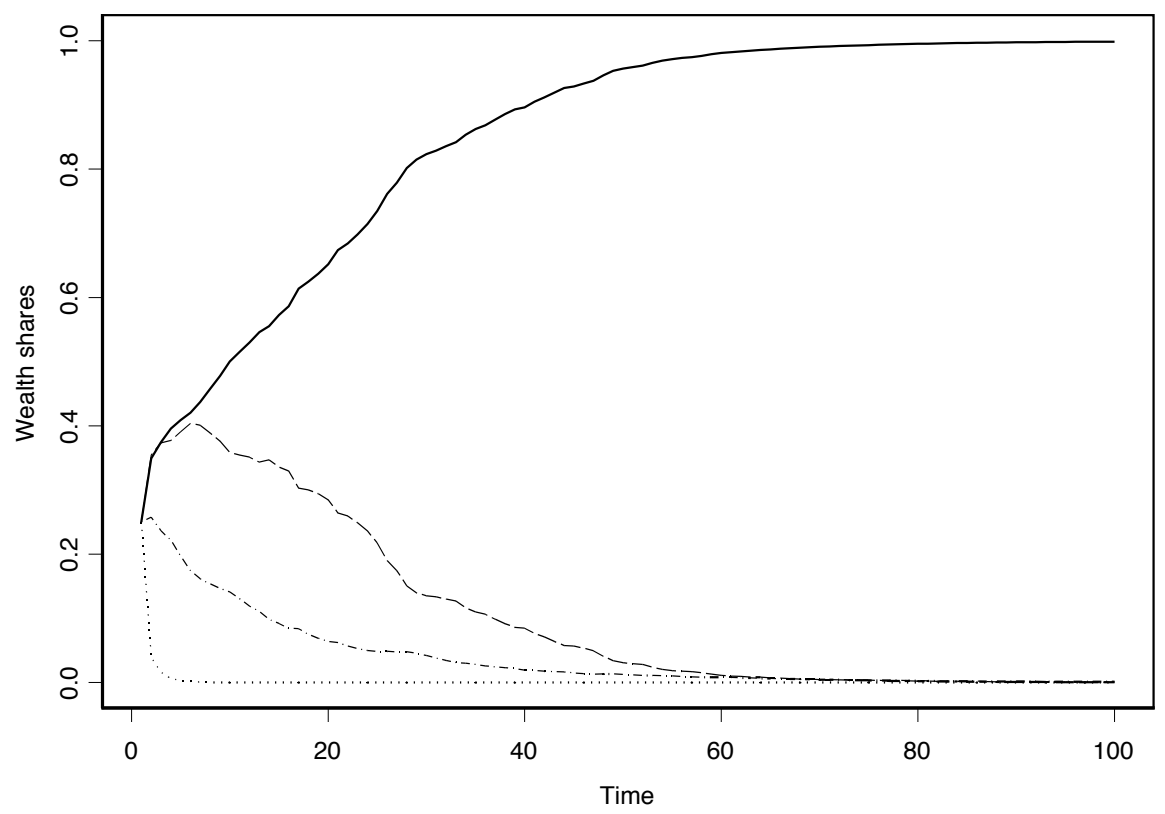

(b)

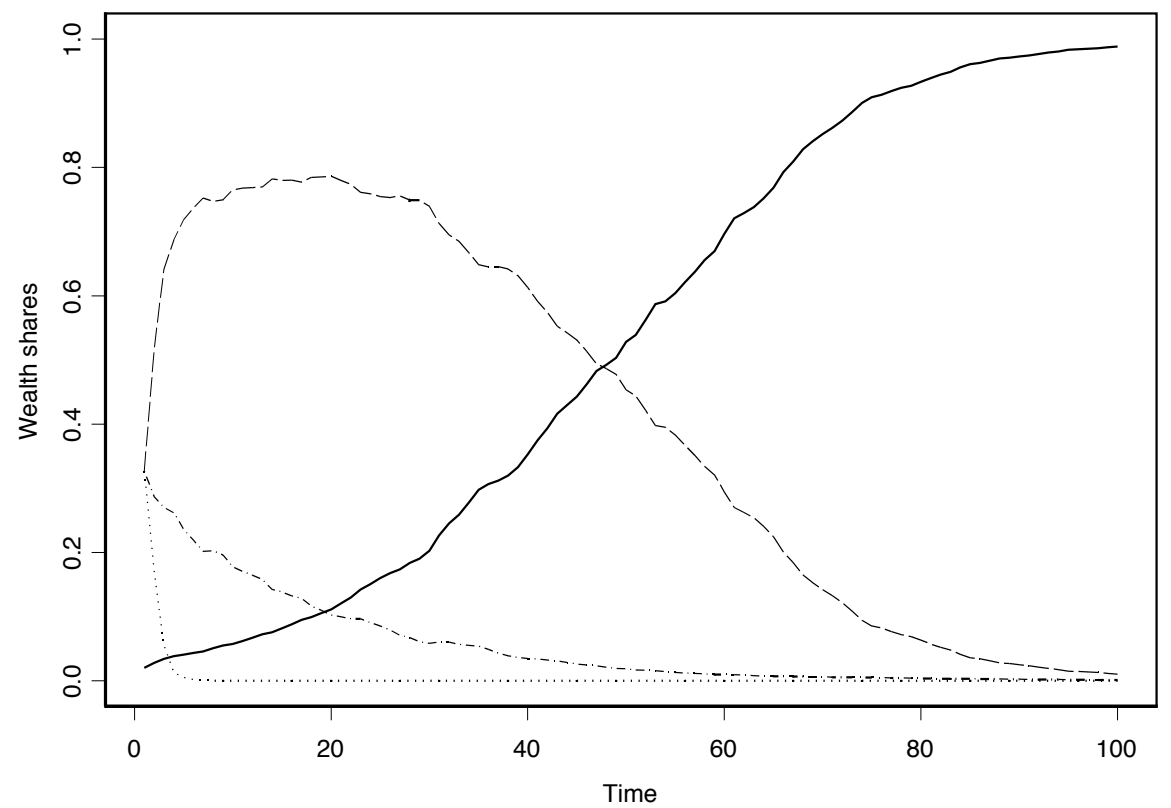

Figure 1: Evolution of market shares, for the $\lambda^{\star}$ strategy of Theorem 2 (full line), the strategy $\underline{\lambda}$ (dotted line), the risk free strategy (dashed-dotted line), and a randomly chosen strategy in $(0, \underline{\lambda})$ (dashed line). In figure (a) all investors have the same initial wealth, while in figure (b) the strategy $\lambda^{\star}$ initially possesses only the $2 \%$ of the market capital. 


\section{References}

Arnold, L. (1998): Random Dynamical Systems. Springer Verlag, Berlin.

Artzner, P., F. Delbaen, J.-M. Eber, and D. Heath (1997): "Thinking Coherently," Risk, 10(11), 68-71.

Blume, L., And D. EAsley (1992): "Evolution and Market Behavior," Journal of Economic Theory, 58(1), 9-40.

(2002): "If You're So Smart, Why Aren't You Rich? Belief Selection in Complete and Incomplete Markets," Working paper, Department of Economics, Cornell University.

Breiman, L. (1961): "Optimal Gambling Systems For Favorable Games," in Fourth Berkeley Symposium on Mathematical Statistic and Probability, vol. 1, pp. 65-78, Berkeley. University of California Press.

Browne, S. (1997): "Survival and Growth with a Liability: Optimal Portfolio Strategies in Continuous Time," Mathematics of Operation Research, 22(2), 468-493.

(1999): "Reaching Goals by a Deadline: Digital Options and Continuous-Time Active Portfolio Management," Advances in Applied Probability, 31(2), 551-577.

Carino, D., D. Myers, and W. Ziemba (1998): "Concepts, Technical Issues, and Uses of the Russel-Yasuda Kasai Financial Planning Model," Operations Research, 46, 449-462.

Carino, D., And W. Ziemba (1998): "Formulation of the Russel-Yasuda Kasi Financial Planning Model," Operations Research, 46, 433-449.

Ceccarelli, S. (2002): "Insolvency Risk in the Italian Non-Life Insurance Companies. An Empirical Analysis Based on a Cash Flow Simulation Model," Working paper, Supervisory Commission on Pension Funds (COVIP), Rome.

Davis, P. (2001): "Portfolio Regulation of Life Insurance Companies and Pension Funds," Working paper No. PI-0101, The Pensions Institute, Birkbeck College.

Embrechts, P. (1996): "Actuarial Versus Financial Pricing of Insurance," Working paper No. 96-17, The Wharton School, University of Pennsylvania.

Embrechts, P., E. De Giorgi, F. Lindskog, and A. McNeil (2002): "Audit of the Swiss Re Group Risk Model," Unpublished report for Swiss Re.

Evstigineev, I., T. Hens, and K. Schenk-Hoppé (2003): "Evolutionary Stable Stock Markets," Working paper No. 84, NCCR FINRISK Working paper series. 
Evstigneev, I., T. Hens, And K. Schenk-Hoppé (2002): "Market Selection of Financial Trading Strategies: Global Stability," Mathematical Finance, 12(4), 329-339.

Feller, W. (1971): An Introduction to Probability Theory and Its Applications, vol. 2. Wiley, New York.

Hakansson, N., And W. Ziemba (1995): "Capital Growth Theory," in Handbooks in Operations Research and Management Science, ed. by R. Jarrow, V. Maksimovic, and W. Ziemba, vol. 9, pp. 65-86. Elsevier, Amsterdam.

Heath, D., S. Orey, W. Pestien, and W. Sudderth (1987): "Minimizing or Maximizing the Expected Time to Reach Zero," SIAM Journal of Control and Optimization, 25(1), 195-205.

Hens, T., And K. Schenk-Hoppé (2002a): "Evolution of Portfolio Rules in Incomplete Markets," Working paper No. 74, Institute for Empirical Research in Economics, University of Zurich.

Hens, T., And K. Schenk-Hoppé (2002b): "Markets Do Not Select For a Liquidity Preference as Behaviour Towards Risk," Working paper No. 139, Institute for Empirical Research in Economics, University of Zurich.

Horn, R., and C. Johnson (1985): Matrix Analysis. Cambridge University Press, Cambridge UK.

Karatzas, I., And S. Shreve (1998): Methods of Mathematical Finance. Springer-Verlag, New York.

Kelly, J. (1956): "A New Interpretation of Information Rate," Bell System Technical Journal, 35, 917-926.

Leippold, M., P. VAnini, and F. Trojani (2003): "Equilibrium Impact of Value-at-Risk Regulation," Working paper, University of Zurich and Università della Svizzera Italiana.

Liu, J., F. A. Longstaff, and J. Pan (2003): "Dynamic Asset Allocation With Event Risk," Journal of Finance, 58(1), 231-259.

LucAs, R. (1978): "Asset Prices in an Exchange Economy," Econometrica, 46(6), 14291445.

Norberg, R., And B. Sundt (1985): "Draft on a System for Solvency Control in Non-Life Insurance," ASTIN Bulletin, 15(2), 150-169.

Pestien, V., And W. Sudderth (1985): "Continuous-Time Red and Black: How to Control a Diffusion to a Goal," Mathematics of Operations Research, 10(4), 599-611. 
Sandroni, A. (2000): "Do Markets Favor Agents Able to Make Accurate Predictions?," Econometrica, 68(6), 1303-1341.

SchniePer, R. (1993): "The Insurance of Catastrophe Risks," SCOR Notes, April 1993.

Thorp, E. (1971): "Portfolio Choice and the Kelly Criterion," in Stochastic Models in Finance, ed. by W. Ziemba, and R. Vickson, pp. 599-619. Academic Press, New York.

Zhao, Y., And W. Ziemba (2000): "A Dynamic Asset Allocation Model with Downside Risk Control," The Journal of Risk, 3(1), 91-113.

Ziemba, W. (2002): "The Capital Growth Theory of Investment: Part I," Willmott Magazine, December, 16-18.

\section{Appendix}

\subsection{Existence and uniqueness of $\delta_{t}^{i}$ and $P_{t+1}$}

For the sake of simplicity we drop the index $t$ from the notation of equations (7) and (8). Using the expression (7) for $\delta^{i}$ in (8), we obtain

$$
P=\mu+\sum_{i}\left(\frac{\sigma \theta^{i}}{\mu+\sigma \theta^{i}-P}-1\right) \alpha^{i} w^{i},
$$

where $\theta^{i}=F^{-1}\left(1-\epsilon^{i}\right)$. Let $f:\left[0, \min _{i}\left\{\mu+\sigma \theta_{i}\right\}\right) \rightarrow \mathbb{R}$ be defined by $f(P)=\mu+$ $\sum_{i}\left(\frac{\sigma \theta^{i}}{\mu+\sigma \theta^{i}-P}-1\right) \alpha^{i} w^{i} . f$ is well defined on $\left[0, \min _{i}\left\{\mu+\sigma \theta_{i}\right\}\right)$ and continuous differentiable, with $f^{\prime}(P)=\sum_{i} \frac{\sigma \theta^{i}}{\left(\mu+\sigma \theta^{i}-P\right)^{2}} \alpha^{i} w^{i}>0, f^{\prime \prime}(P)=\sum_{i} \frac{2 \sigma \theta^{i}}{\left(\mu+\sigma \theta^{i}-P\right)^{3}} \alpha^{i} w^{i}>0$, i.e. $f$ is strictly increasing and convex. Moreover, $f(P) \nearrow \infty$ as $P \nearrow \min _{i}\left\{\mu+\sigma \theta_{i}\right\}$ and

$$
\begin{aligned}
f(0) & =\mu+\sum_{i}\left(\frac{\sigma \theta^{i}}{\mu+\sigma \theta^{i}}-1\right) \alpha^{i} w^{i}=\mu-\mu \sum_{i} \frac{\alpha^{i} w^{i}}{\mu+\sigma \theta^{i}} \\
& =\mu-\mu \underbrace{\left.\sum_{i} \delta^{i}\right|_{P=0}}_{=1}=0 \\
f^{\prime}(0) & =\sum_{i} \frac{\sigma \theta^{i} \alpha^{i} w^{i}}{\left(\mu+\sigma \theta^{i}\right)^{2}} \leq \max _{i}\left\{\frac{\sigma \theta^{i}}{\mu+\sigma \theta^{i}}\right\} \underbrace{\sum_{i} \frac{\alpha^{i} w^{i}}{\mu+\sigma \theta^{i}}}_{=1}<1,
\end{aligned}
$$

since $\mu>0$. Thus $f(P) \geq 0$ and it possesses exactly two fixed points: $P=0$ and $P^{\star} \in$ $\left(0, \min _{i}\left\{\mu+\sigma \theta_{i}\right\}\right)$. Therefore, there is a unique premium $P^{*}>0$ which satisfies equations (7) and (8). Moreover, by equation (7), $\delta^{i}$ is also uniquely defined for all $i$. 


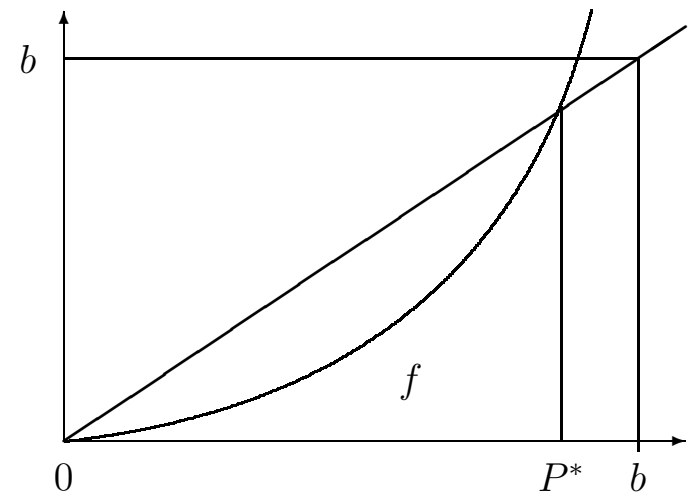

Figure 2: Proof of the existence and uniqueness of equilibrium insurance premium. The demand function $f(P)$ has one strictly positive fixed point $P^{*}$.

\subsection{Derivation of the wealth dynamics}

$$
\begin{aligned}
w_{t+1}^{i}= & A_{t+1}^{i}+\frac{B_{t}^{i}}{1-\sum_{j} \lambda_{t+1}^{j} B_{t}^{j}} \sum_{j} \lambda_{t+1}^{j} A_{t}^{j} \\
= & A_{t+1}^{i}+\frac{\lambda_{t}^{i} w_{t}^{i}}{\sum_{j} \lambda_{t}^{j} w_{t}^{j}-\sum_{j} \lambda_{t+1}^{j} \lambda_{t}^{j} w_{t}^{j}} \sum_{j} \lambda_{t+1}^{j} A_{t}^{j} \\
= & w_{t}^{i}\left[R\left(1-\lambda_{t}^{i}\right)+\frac{\lambda_{t}^{i}}{\boldsymbol{\lambda}_{t}^{T} \mathbf{w}_{t}} d_{t+1} W_{t}-\left(\eta_{t+1}-\beta+\alpha\right)\right. \\
& \left.\quad+\lambda_{t}^{i} \frac{\sum_{j} w_{t}^{j} \lambda_{t+1}^{j}\left(R\left(1-\lambda_{t}^{j}\right)+\frac{\lambda_{t}^{j}}{\boldsymbol{\lambda}_{t}^{T} \mathbf{w}_{t}} d_{t+1} W_{t}-\left(\eta_{t+1}-\beta+\alpha\right)\right)}{\sum_{j}\left(1-\lambda_{t+1}^{j}\right) \lambda_{t}^{j} w_{t}^{j}}\right]
\end{aligned}
$$


Thus

$$
\begin{aligned}
w_{t+1}^{i}= & \frac{w_{t}^{i}}{\boldsymbol{\lambda}_{t}^{T} \mathbf{w}_{t}}\left[R\left(1-\lambda_{t}^{i}\right) \boldsymbol{\lambda}_{t}^{T} \mathbf{w}_{t}+\lambda_{t}^{i} d_{t+1} W_{t}-\left(\eta_{t+1}-\beta+\alpha\right) \boldsymbol{\lambda}_{t}^{T} \mathbf{w}_{t}\right. \\
& \left.\quad+\lambda_{t}^{i} \frac{\sum_{j} w_{t}^{j} \lambda_{t+1}^{j}\left(R\left(1-\lambda_{t}^{j}\right) \boldsymbol{\lambda}_{t}^{T} \mathbf{w}_{t}+\lambda_{t}^{j} d_{t+1} W_{t}-\left(\eta_{t+1}-\beta+\alpha\right) \boldsymbol{\lambda}_{t}^{T} \mathbf{w}_{t}\right)}{\sum_{j}\left(1-\lambda_{t+1}^{j}\right) \lambda_{t}^{j} w_{t}^{j}}\right] \\
= & \frac{w_{t}^{i}}{\boldsymbol{\lambda}_{t}^{T} \mathbf{w}_{t}} \frac{1}{\sum_{j}\left(1-\lambda_{t+1}^{j}\right) \lambda_{t}^{j} w_{t}^{j}} \times \\
& {\left[\sum_{j}\left(1-\lambda_{t+1}^{j}\right) \lambda_{t}^{j} w_{t}^{j}\left(R\left(1-\lambda_{t}^{i}\right) \boldsymbol{\lambda}_{t}^{T} \mathbf{w}_{t}+\lambda_{t}^{i} d_{t+1} W_{t}-\left(\eta_{t+1}-\beta+\alpha\right) \boldsymbol{\lambda}_{t}^{T} \mathbf{w}_{t}\right)\right.} \\
& \left.\quad+\lambda_{t}^{i}\left(\sum_{j} w_{t}^{j} \lambda_{t+1}^{j}\left(R\left(1-\lambda_{t}^{j}\right) \boldsymbol{\lambda}_{t}^{T} \mathbf{w}_{t}+\lambda_{t}^{j} d_{t+1} W_{t}-\left(\eta_{t+1}-\beta+\alpha\right) \boldsymbol{\lambda}_{t}^{T} \mathbf{w}_{t}\right)\right)\right] \\
= & \frac{w_{t}^{i}}{\sum_{j}\left(1-\lambda_{t+1}^{j}\right) \lambda_{t}^{j} w_{t}^{j}} \times \\
& {\left[d_{t+1} W_{t} \lambda_{t}^{i}+\left[R\left(1-\lambda_{t}^{i}\right)-\left(\eta_{t+1}-\beta+\alpha\right)\right]\left(\boldsymbol{\lambda}_{t}^{T} \mathbf{w}_{t}+\sum_{j}\left(\lambda_{t}^{i}-\lambda_{t}^{j}\right) \lambda_{t+1}^{j} w_{t}^{j}\right)\right] }
\end{aligned}
$$

and therefore

$$
\begin{aligned}
w_{t+1} & =\frac{w_{t}^{i}}{\sum_{j}\left(1-\lambda_{t+1}^{j}\right) \lambda_{t}^{j} w_{t}^{j}} \times \\
& {\left[d_{t+1} W_{t} \lambda_{t}^{i}+\left[R\left(\underline{\lambda}-\lambda_{t}^{i}\right)+\left(\bar{\eta}-\eta_{t+1}\right)\right]\left(\lambda_{t}^{T} \mathbf{w}_{t}+\sum_{j \neq i}\left(\lambda_{t}^{i}-\lambda_{t}^{j}\right) \lambda_{t+1}^{j} w_{t}^{j}\right)\right] . }
\end{aligned}
$$

We use that

$$
\underline{\lambda}=\frac{R-\bar{\eta}+\beta-\alpha}{R} \Longleftrightarrow R+\beta-\alpha=R \underline{\lambda}+\bar{\eta} .
$$

